\title{
Trade-credit modeling for deteriorating item inventory system with preservation technology under random planning horizon
}

\author{
DIPANA JYOTI MOHANTY ${ }^{1}$, RAVI SHANKAR KUMAR ${ }^{2}$ and A GOSWAMI ${ }^{1, *_{0}}$ \\ ${ }^{1}$ Department of Mathematics, Indian Institute of Technology, Kharagpur 721302, India \\ ${ }^{2}$ National Institute of Technology Agartala, Agartala 799046, India \\ e-mail: dipanajyoti80@gmail.com; ravikhushi412@yahoo.co.in; goswami@maths.iitkgp.ernet.in
}

MS received 7 June 2017; revised 10 August 2017; accepted 10 August 2017; published online 23 March 2018

\begin{abstract}
This paper deals with a stochastic deteriorating item inventory model with preservation technology and trade-credit finance. The planning horizon of many seasonal or fashionable items stochastically varies to some extent. On the other side, the demand of such items increases in initial phase followed by a constant. This type of demand pattern can be modeled as ramp-type demand. Deterioration of such products is a characteristic, which can be reduced by making the investment on latest equipment and technology. Consumption of such items within shelf life prevents to deterioration, which can be achieved by bulk sale. In order to stimulate the selling, trade-credit policy is also considered here. In these regards, this study examines the joint effect of preservation technology investment and trade-credit policy, wherein shortage is allowed and mixture of partial backlog and lost sales. Six cases may arise depending upon three parameters of time $\mu$ (demand increases up to $\mu$ ), $T$ (when on-hand inventory reaches to zero) and $M$ (trade-credit period). The mathematical models are mainly categorized in two cases: (i) $\mu \leq M$ and (ii) $\mu>M$. The model is illustrated through numerical experiments, sensitivity analysis, and graphical representation.
\end{abstract}

Keywords. Inventory; stochastic review period; deterioration; preservation technology; trade-credit policy; partial backlogging.

\section{Introduction}

Planning horizon of many seasonal and fashionable items, in general, stochastically fluctuate in some extend. As for example, in India, winter starts with November and ends with February. But its duration is not always fixed. A little stochastic variability can be easily noticed over the years. Thus, planning horizon of seasonal products such as fruits, clothes, food grains, etc. is a random variable instead of a fixed deterministic constant. Demand for such products, in general, increases in initial phase followed by a constant. This type of demand pattern is referred as ramp-type in the literature [1]. This paper is intended to develop an deteriorating inventory model for ramp-type demand in stochastic framework, wherein deterioration rate is reduced through some technology called preservation technology (PT). Consumption of such item within shelf life is another way to avoid the deterioration. In order to stimulate the selling, we incorporate trade-credit finance in form of permissible delay in payment.

Demand is one of the most important key parameter of inventory/supply chain management. Multifariousness of deterministic demand such as uniform, linear, time

*For correspondence dependent and ramp-type has been modeled by many authors ([2, 3]). Hill [1] first considered ramp-type demand into an inventory problem and derived an EOQ formula. After that many authors have modeled it for deteriorating items' inventory problem such as [4-12] and [13].

Deterioration is the phenomena of vaporization, damage, spoilage, dryness, etc. that decrease the usefulness of the product from the original one [14]. Deterioration rate significantly influences inventory, sales, and order quantities, especially for fast deteriorating products, which finally results in higher total relevant cost and lower demand rate [15]. Ouyang et al [16] suggested that deterioration rate can be reduced by improving the storage facility, which finally reduces the overall relevant cost. In recent years, many enterprises invest on types of equipment such as refrigeration, drying and vacuum technology to reduce the deterioration rate and to extend the product expiration date [17]. Zauberman et al [18] developed a method for color retention of Litchi fruits with $\mathrm{SO}_{2}$ fumigation. In order to reduce the deterioration rate and to extend the expiration date of the product, preservation technologies (PT) like procedural changes and specialized equipment acquisition have been mathematically modeled by many researchers. The sensitivity analysis on numerical studies by [19] said that a lower deterioration rate is beneficial from the economic 
viewpoint. Geetha and Uthayakumar [20] also justified [19] observation. Hsu et al [17] proposed a deteriorating inventory model considering constants deterioration and demand rates, wherein PT also included. They determine the retailer's replenishment and PT strategies in order to maximize the retailer's profit. Moreover, that model also considered fixed cost of PT which was independent of replenishment cycle length. Dye and Hsieh [14] extended [17] by considering time-varying deterioration and backlogging rates and replenishment cycle length dependent PT cost. Lee and Dye [21] extended [17] by considering stockdependent demand rate and replenishment cycle length dependent PT cost. Hsieh and Dye [22] developed a production-inventory model with controllable deterioration rate by considering time-dependent demand rate and PT cost as a decision variable in conjunction with production policy. Dye [23] studied PT investment and inventory decisions for a retailer's non-instantaneous deteriorating items, and through a sensitivity analysis delineated its dependency on cost parameters. Mishra et al [24] developed a deteriorating inventory model for price and stock dependent demand by employing PT. In recent years, deteriorating inventory model employing PT under different business scenarios such as optimal pricing, service and demand patterns, has been modeled by [25, 26] and [27].

Trade-credit modeling in deteriorating inventory has received considerable attention in recent business scenarios, especially, for items having the finite shelf life. Tradecredit in term of permissible delay in payment is a time span between purchasing and payment, which is generally offered by an upper-stair partner to lower-stair partner in a supply chain (SC). The lower-stair SC partner can earn interest on the money during the trade-credit period and may pay interest for delayed settlement after the end of the credit period. Goyal [28] derived retailer's EOQ (Economic Order Quantity) when a supplier offers a permissible delay in payment. Aggarwal and Jaggi [29] extended [28] by incorporating deterioration. Chang et al [30] developed an EOQ model by considering order quantity linked trade-credit period and deterioration. Deteriorating inventory models with the permissible delay in payment under different business environment have been developed by [16] and [19]. Ouyang et al [31] extended [30] by considering an order quantity dependent partially permissible delay in payment. Skouri et al [8] modeled ramp-type demand pattern in a deteriorating inventory problem by incorporating PT. Dye and Hsieh [32] developed a deteriorating inventory model for finite time horizon, wherein PT and trade-credit are taken into account. Yang et al [33] extended [32] by considering time and trade-credit period dependent demand and risk. Chen and Teng [34] enhanced deteriorating inventory model under trade-credit finance by considering expiration date and discount cash flow analysis. Tiwari et al [35] addressed a two-warehouse inventory model by integrating the issues of trade-credit, non-instantaneous deterioration, and inflation, wherein shortages are allowed. Recently, Tsao and Sheen [36] extended [23] by addressing the problem of joint location and PT investment decision making for non-instantaneous deteriorating items under trade-credit. All the discussed trade-credit modeling problems are developed by considering deterministic constant planning horizon. But as discussed at beginning of this section, in many cases planning horizon varies randomly.

We have considered random time horizon, instead of deterministic time horizon, which is the major contribution of the paper.

As for our knowledge and an evidence of the literature survey, no inventory model has been developed in stochastic framework considering time dependent ramptype demand, preservation technology and trade-credit finance. Stochastic variability in planning horizon of seasonal and fashionable product is worthwhile, because it depends upon many environmental and other factors. Thus, purpose of this study is twofold: (1) Extend the deterministic inventory model for ramp-type demand in stochastic environment by considering planning horizon as a random variable. (2) To develop a methodology to account the opportunity cost and opportunity gain for stochastic period. The rest of the paper is organized as follows: Section 2 lists all the notations, and describes the assumption made in this study. Section 3 mathematically formulates the model under the assumptions made in section 2. Section 4 develops a methodology to account the opportunity gain and opportunity cost for stochastic period. Solution methodology is provided in sections 5 and 6 . Section 7 provides some numerical illustrations in support of theoretical discussion. This section also finds sensitivity analysis by changing the value of key parameters, and through which delineates managerial insights. Section 8 is concluding remark section which also provides future direction of this study.

\section{Notations and assumptions}

The following notations are used throughout the paper.

\subsection{Notation}

\section{Parameters:}

$x \quad$ random scheduling period with probability density function $f(x)$, where $\left(x_{\min } \leq x \leq x_{\max }\right)$

$M$ permissible delay in payment

$\theta$ deterioration rate

$s \quad$ per unit selling price

$p \quad$ per unit purchasing price

A ordering cost

$h$ inventory holding cost per product per unit time 
$d$ disposal cost per product

$b$ back-logging cost per product per unit time

$l$ the goodwill cost due to lost sale per unit product

$e \quad$ interest earned per product per unit time

$c$ interest charged per product per unit time

$\alpha$ parameter of demand function

$\mu \quad$ time within which ramp-type demand increases

$\beta$ percentage of partially back order

$\delta \quad$ parameter of exponential distribution

\section{Decision variables:}

$T$ time to finish the on-hand inventory after replenishment

$\xi$ the preservation technology cost per unit time

\section{Dependant variables:}

$\bar{I}_{c} \quad$ expected inventory for which interest paid

$\bar{I}_{e} \quad$ expected inventory for which interest earned

$\bar{P} \quad$ expected review period

$\bar{D}$ expected deteriorating items

$\bar{Q}$ expected order quantity

$\bar{I}$ expected inventory

$\bar{B} \quad$ expected shortage

$\bar{L} \quad$ expected lost sale

$S$ maximum inventory level.

\subsection{Assumptions}

While developing the proposed model, the following assumptions are made.

1. Demand rate is time dependent ramp-type which is defined as

$$
r(t)= \begin{cases}\alpha t, & t \leq \mu \\ \alpha \mu, & t>\mu\end{cases}
$$

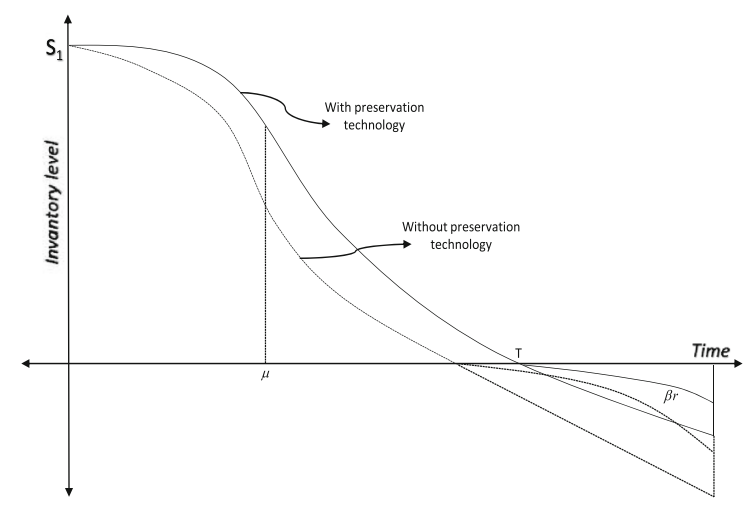

(a) $\mu \leq T$ where $\mu>0$ and $\alpha>0$.

2. Shortage is allowed, and is mixture of partially backlogging and lost sales. The backlogging quantity is delivered at the end of the period.

3. A single period inventory system is considered, wherein planning period is a random variable.

4. The original rate of deterioration is constant, and is $\theta$ (a fraction of on-hand inventory), where $0 \leq \theta<1$.

5. The deterioration rate is reduced through investment in preservation technology $\xi$. The proportion of reduced deterioration rate is $m(\xi)=\theta\left(1-e^{-\gamma \xi}\right)$, where $\gamma>0$, $\xi>0$, and $\gamma$ is the simulation coefficient representing the percentage increase in $m(\xi)$ per dollar increase in $\xi$, is a continuous, concave, increasing function of retailer's capital investment, where $m(0)=0$ and $\lim _{\xi \rightarrow \infty} m(\xi)=\theta$. Note that $m^{\prime}(\xi)>0$ and $m^{\prime \prime}(\xi)<0$ imply the diminishing marginal productivity of capital.

6. Repairing or replacement for the deteriorated item is not permissible.

7. A trade-credit period $M$ is offered to the retailer to settle the purchasing amount. The sale revenue of the period $[0, M]$ is deposited in an interest bearing account. If $M<T$, then the retailer has also to pay the interest on the investment of remaining inventory for the period $[M, T]$.

\section{Mathematical formulation}

In this section, we mathematically model the proposed inventory control problem. The inventory fluctuation over time is shown in figure 1. The figure also delineates the effect of PT. The smooth curve indicates inventory level when PT has applied while the dotted curve for without PT. At the beginning of the period, replenishment is made which bring the inventory level up to $S$. After that, it is depleted due to demand and deterioration up to $T$. At that time inventory level reaches at zero, then shortage starts and continues up to the end of the period. A fraction of the

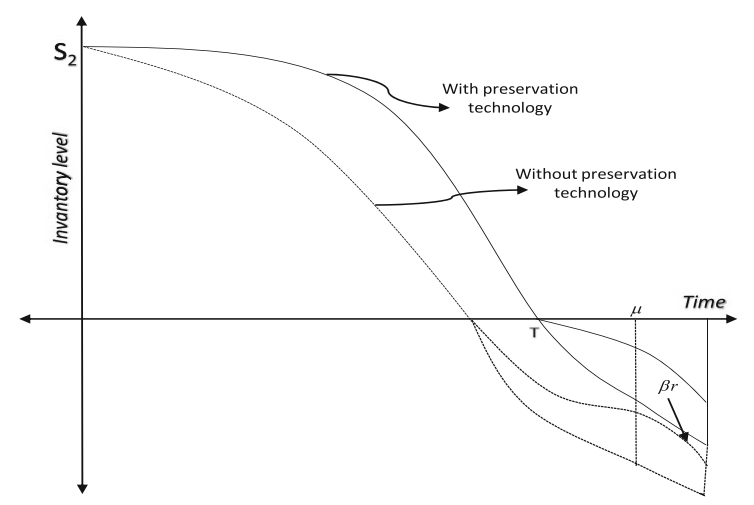

(b) $T<\mu$

Figure 1. Inventory positions with PT and without PT. 
shortage is backlogged and remaining lost sale. As shown in the figure, inventory fluctuation depends upon values of $\mu$ and $T$. Hence, we consider two cases: (1) $\mu \leq T$ and (2) $\mu>T$.

\subsection{Case I. When $\mu \leq T$}

In this case, shortage starts before $\mu$ and continues up to the end of the period as shown in figure 1(a). The inventory level is governed by the following differential equation.

$$
\left\{\begin{array}{lc}
\frac{d i}{d t}+(\theta-m(\xi)) i=-\alpha t, & 0 \leq t \leq \mu ; \\
\frac{d i}{d t}+(\theta-m(\xi)) i=-\alpha \mu, & t \geq \mu
\end{array}\right.
$$

with initial condition

$$
i=\left\{\begin{aligned}
i_{1}(t)=-\frac{\alpha}{\theta-m(\xi)}\left(t-\frac{1}{\theta-m(\xi)}\right) & \\
+\left(S_{1}-\frac{\alpha}{(\theta-m(\xi))^{2}}\right) e^{-(\theta-m(\xi)) t}, & 0 \leq t \leq \mu ; \\
i_{2}(t)=\frac{\alpha \mu}{\theta-m(\xi)}\left(e^{-(\theta-m(\xi))(t-T)}-1\right), & t \geq \mu .
\end{aligned}\right.
$$

$i(T)=0$ gives

$$
S_{1}=\frac{\alpha}{\theta-m(\xi)}\left(\mu e^{(\theta-m(\xi)) T}+\frac{1-e^{(\theta-m(\xi)) \mu}}{\theta-m(\xi)}\right) .
$$

Total accumulated inventory over the random interval $[0, x]$ is:

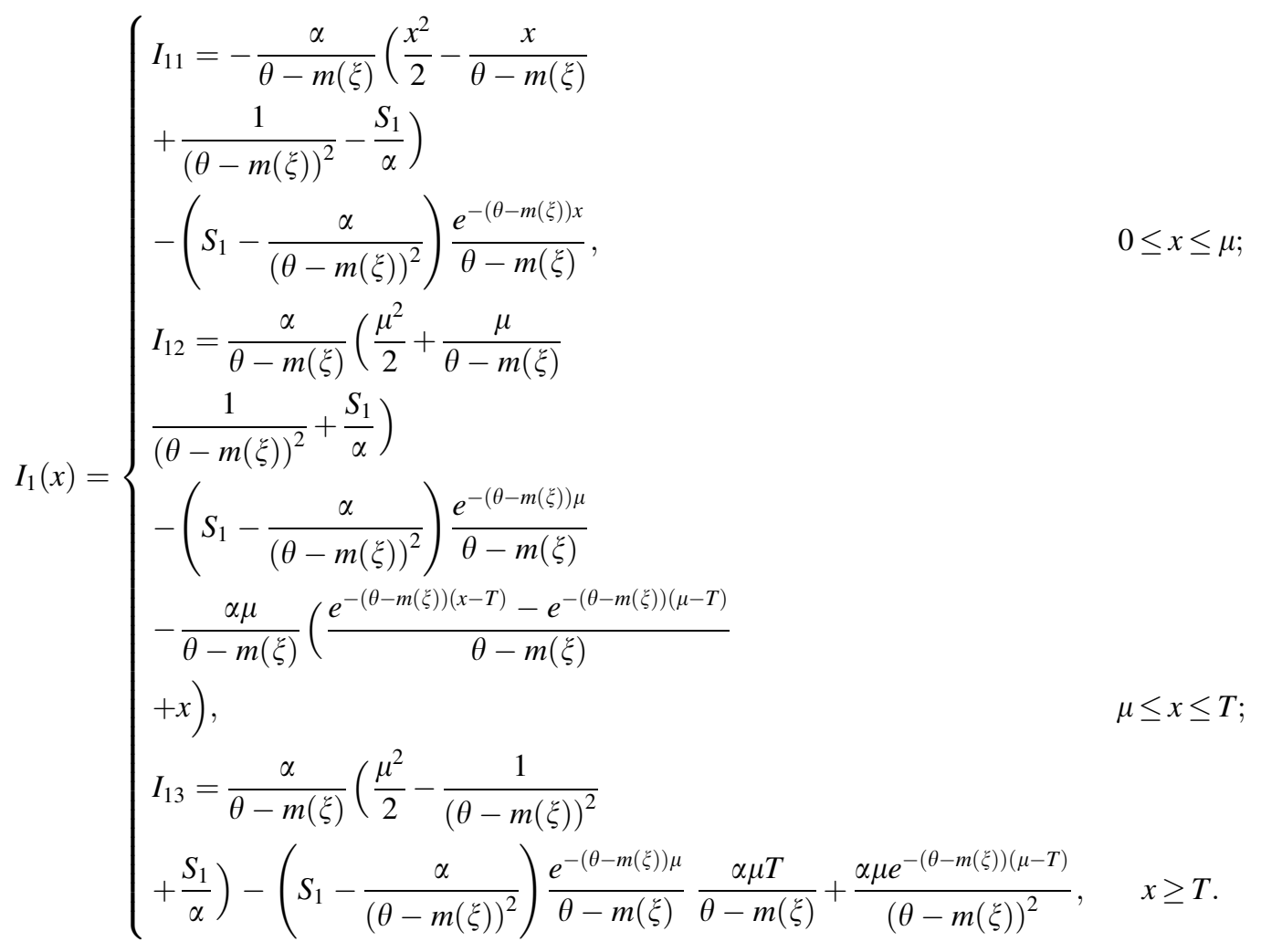

$$
i(0)=S_{1}
$$

and boundary condition

$$
i(T)=0 .
$$

Eqs. (3.1) and (3.2) give
The planning period $x$ is a random variable, consequently, inventory level, order quantity, back-order quantity, etc. are random variables. Therefore we obtain the expected values of these quantities as follows: 


$$
\begin{aligned}
\overline{Q_{1}}=E\left(Q_{1}\right)= & \int_{x_{\min }}^{\mu}\left(S_{1}-i_{1}(x)\right) f(x) d x \\
& +\int_{\mu}^{T}\left(S_{1}-i_{2}(x)\right) f(x) d x \\
& +\int_{T}^{x_{\max }}\left(S_{1}+\alpha \mu \beta(x-T)\right) f(x) d x \\
\overline{I_{1}}=E\left(I_{1}\right)= & \int_{x_{\min }}^{\mu} I_{11} f(x) d x+\int_{\mu}^{T} I_{12} f(x) d x \\
& +\int_{T}^{x_{\max }} I_{13} f(x) d x, \\
\overline{D_{1}}=E\left(D_{1}\right)= & \int_{x_{\min }}^{\mu}\left(S_{1}-i_{1}(x)-\alpha \frac{x^{2}}{2}\right) f(x) d x \\
& +\int_{\mu}^{T}\left(S_{1}-i_{2}(x)+\alpha \mu \frac{\mu-2 x}{2}\right) f(x) d x \\
& +\int_{T}^{x_{\max }}\left(S_{1}+\alpha \mu \frac{\mu-2 T}{2}\right) f(x) d x
\end{aligned}
$$

$$
\begin{aligned}
\overline{V_{1}}=\overline{Q_{1}}-\overline{D_{1}}= & \frac{\alpha}{2} \int_{x_{\min }}^{\mu} x^{2} f(x) d x \\
& +\alpha \mu \int_{\mu}^{T}\left(x-\frac{\mu}{2}\right) f(x) d x \\
& +\alpha \mu \int_{T}^{x_{\max }}\left(\left(T-\frac{\mu}{2}\right)+\beta(x-T)\right) f(x) d x,
\end{aligned}
$$

$$
\begin{gathered}
\overline{B_{1}}=E\left(B_{1}\right)=\alpha \mu \beta \int_{T}^{x_{\max }} \frac{(x-T)^{2}}{2} f(x) d x, \\
\overline{L_{1}}=E\left(L_{1}\right)=\alpha \mu(1-\beta) \int_{T}^{x_{\max }}(x-T) f(x) d x, \\
\bar{P}=E(x)=\int_{x_{\min }}^{x_{\max }} x f(x) d x .
\end{gathered}
$$

\subsection{Case II. When $\mu>T$}

As shown in figure 1(b), for this case, shortage starts before $\mu$ and continues up to the end of the random period $x$. The inventory level is governed by the following differential equation.

$$
\frac{d i}{d t}+(\theta-m(\xi)) i=-\alpha t, 0 \leq t \leq T
$$

with initial condition

$$
i(0)=S_{2}
$$

and boundary condition

$$
i(T)=0 .
$$

Eqs. (3.14) and (3.15) give

$$
\begin{aligned}
i= & -\frac{\alpha}{\theta-m(\xi)}\left(t-\frac{1}{\theta-m(\xi)}\right) \\
& +\left(S_{2}-\frac{\alpha}{(\theta-m(\xi))^{2}}\right) e^{-(\theta-m(\xi)) t}, \quad 0 \leq t \leq T .
\end{aligned}
$$

The boundary condition $i(T)=0$ gives

$$
S_{2}=\frac{\alpha}{\theta-m(\xi)}\left(\left(T-\frac{1}{\theta-m(\xi)}\right) e^{(\theta-m(\xi)) T}+\frac{1}{\theta-m(\xi)}\right) .
$$

The random accumulated inventory over the random interval $[0, x]$ is:

$$
I_{2}(x)=\left\{\begin{aligned}
& I_{21}=-\frac{\alpha}{\theta-m(\xi)}\left(\frac{x^{2}}{2}-\frac{x}{\theta-m(\xi)}\right. \\
&\left.+\frac{1}{(\theta-m(\xi))^{2}}-\frac{S_{2}}{\alpha}\right) \\
&-\left(S_{2}-\frac{\alpha}{(\theta-m(\xi))^{2}}\right) \frac{e^{-(\theta-m(\xi)) x}}{\theta-m(\xi)}, \quad 0 \leq x \leq T ; \\
& I_{22}=-\frac{\alpha}{\theta-m(\xi)}\left(\frac{T^{2}}{2}-\frac{T}{\theta-m(\xi)}\right. \\
&\left.\quad+\frac{1}{(\theta-m(\xi))^{2}}-\frac{S_{2}}{\alpha}\right) \\
&-\left(S_{2}-\frac{\alpha}{(\theta-m(\xi))^{2}}\right) \frac{e^{-(\theta-m(\xi)) T}}{\theta-m(\xi)}, \quad x \geq T .
\end{aligned}\right.
$$

The expected values of order quantity, inventory level, deterioration, sale quantity, backlogging quantity and lost sale are as follow:

$$
\begin{aligned}
\overline{Q_{2}}=E\left(Q_{2}\right)= & \int_{x_{\min }}^{T}\left(S_{2}-i\right) f(x) d x \\
& +\int_{T}^{\mu}\left(S_{2}+\frac{\alpha \beta}{2}(x-T)^{2}\right) f(x) d x \\
& +\int_{\mu}^{x_{\max }}\left(S_{2}+\frac{\alpha \beta}{2}(\mu-T)^{2}\right. \\
& +\alpha \mu \beta(x-\mu)) f(x) d x,
\end{aligned}
$$

$$
\begin{aligned}
\overline{I_{2}}=E\left(I_{2}\right)= & \int_{x_{\min }}^{T} I_{21} f(x) d x \\
& +\int_{T}^{x_{\max }} I_{22} f(x) d x,
\end{aligned}
$$




$$
\begin{aligned}
\overline{D_{2}}=E\left(D_{2}\right)= & \int_{x_{\min }}^{T}\left(S_{2}-i-\frac{\alpha x^{2}}{2}\right) f(x) d x \\
& +\int_{T}^{x_{\max }}\left(S_{2}-\frac{\alpha T^{2}}{2}\right) f(x) d x \\
\overline{V_{2}}=\overline{Q_{2}}-\overline{D_{2}}= & \frac{\alpha}{2} \int_{x_{\min }}^{T} x^{2} f(x) d x \\
& +\frac{\alpha}{2} \int_{T}^{\mu}\left(T^{2}+\beta(x-T)^{2}\right) f(x) d x \\
& +\frac{\alpha}{2} \int_{\mu}^{x_{\max }}\left(T^{2}+\beta(\mu-T)^{2}\right. \\
& +2 \beta \mu(x-\mu)) f(x) d x, \\
\overline{B_{2}}=E\left(B_{2}\right)=\frac{\alpha \beta}{6} & \int_{T}^{\mu}(x-T)^{3} f(x) d x \\
+ & \frac{\alpha \beta}{2} \int_{\mu}^{x_{\max }}\left(\frac{(\mu-T)^{3}}{3}+\mu(x-\mu)^{2}\right) f(x) d x, \\
& +\mu(x-\mu)) f(x) d x . \\
& +\alpha(1-\beta) \int_{\mu}^{x_{\max }}\left(\frac{(\mu-T)^{2}}{2}\right. \\
\overline{L_{2}}=E\left(L_{2}\right)= & \frac{\alpha(1-\beta)}{2} \int_{T}^{\mu}(x-T)^{2} f(x) d x \\
&
\end{aligned}
$$

\section{Opportunity cost and opportunity gain}

As we discussed in introduction section, the retailer is offered a trade-credit period $M$ to settle the purchasing cost. Accumulated sale revenue during this period is deposited in an interest bearing account. If shortage starting time $T$ is such that $T>M$, then the retailer has to pay interest on investment of remaining inventory for the period $[T, M]$. Depending upon the values of $M, \mu$ and $T$, we consider six cases as: (i) $0 \leq M \leq \mu \leq T$, (ii) $0 \leq \mu<M \leq T$, (iii) $0 \leq \mu \leq T<M$, (iv) $0 \leq M \leq T \leq \mu$, (v) $0 \leq T<M \leq \mu$ and (vi) $0 \leq T<\mu<M$. Furthermore, since $\mu$ and $M$ are known parameters, here only $T$ is a decision variable. Depending upon the values of $\mu$ and $T$, we develop two models, namely, Model I: when $M \leq \mu$ and Model II: when $M>\mu$. First we consider six cases of permissible delay:

\subsection{Case $0 \leq M \leq \mu \leq T$}

For this case, the random interest earned and interest paid are obtained as follows:

$$
I_{e 1}=\left\{\begin{array}{cc}
\int_{0}^{x} \int_{0}^{t} \alpha \eta d \eta d t+(M-x) \int_{0}^{x} \alpha t d t, & 0 \leq x \leq M ; \\
\int_{0}^{x} \int_{0}^{t} \alpha \eta d \eta d t, & M \leq x \leq \mu ; \\
\int_{0}^{\mu} \int_{0}^{t} \alpha \eta d \eta d t+\int_{\mu}^{x} \int_{0}^{\mu} \alpha \eta d \eta d t & \\
+\int_{\mu}^{x} \int_{\mu}^{t} \alpha \mu d \eta d t, & \mu \leq x \leq T ; \\
\int_{0}^{\mu} \int_{0}^{t} \alpha \eta d \eta d t+\int_{\mu}^{T} \int_{0}^{\mu} \alpha \eta d \eta d t & \\
+\int_{\mu}^{T} \int_{\mu}^{t} \alpha \mu d \eta d t, & x \geq T ;
\end{array}\right.
$$

and

$$
I_{c 1}= \begin{cases}0, & 0 \leq x \leq M \\ \int_{M}^{x} i_{1}(t) d t, & M \leq x \leq \mu \\ \int_{M}^{\mu} i_{1}(t) d t+\int_{\mu}^{x} i_{2}(t) d t, & \mu \leq x \leq T \\ \int_{M}^{\mu} i_{1}(t) d t+\int_{\mu}^{T} i_{2}(t) d t, & x \geq T\end{cases}
$$

The expected value of interest earned and interest paid are as follows:

$$
\begin{aligned}
I_{e 1}^{-}=E\left(I_{e 1}\right)= & \alpha \int_{x_{\min }}^{M}\left(\frac{M}{2}-\frac{x}{3}\right) x^{2} f(x) d x+\alpha \int_{M}^{\mu} \frac{x^{3}}{6} f(x) d x \\
& +\frac{\alpha \mu}{2} \int_{\mu}^{T}\left(\frac{\mu^{2}}{3}-\mu x+x^{2}\right) f(x) d x \\
& +\frac{\alpha \mu}{2} \int_{T}^{x_{\max }}\left(\frac{\mu^{2}}{3}-\mu T+T^{2}\right) f(x) d x
\end{aligned}
$$

and

$$
\begin{aligned}
I_{c 1}^{-}=E\left(I_{c 1}\right)= & -\frac{\alpha}{\theta-m(\xi)} \int_{M}^{\mu}\left(\left(\frac{x^{2}-M^{2}}{2}-\frac{x-M}{\theta-m(\xi)}\right)\right. \\
& \left.+\left(\frac{S_{1}}{\alpha}-\frac{1}{(\theta-m(\xi))^{2}}\right)\left(e^{-(\theta-m(\xi)) x}-e^{-(\theta-m(\xi)) M}\right)\right) f(x) d x \\
& +\frac{\alpha}{\theta-m(\xi)} \int_{\mu}^{T}\left\{\left(\frac{\mu^{2}+M^{2}}{2}+\frac{\mu-M}{\theta-m(\xi)}\right)\right. \\
& -\left(\frac{S_{1}}{\alpha}-\frac{1}{(\theta-m(\xi))^{2}}\right)\left(e^{-(\theta-m(\xi)) \mu}-e^{-(\theta-m(\xi)) M}\right) \\
& \left.-\mu\left(\frac{e^{-(\theta-m(\xi))(x-T)}-e^{-(\theta-m(\xi))(\mu-T)}}{\theta-m(\xi)}+x\right)\right\} f(x) d x \\
& +\frac{\alpha}{\theta-m(\xi)} \int_{T}^{x_{\max }}\left\{\left(\frac{\mu^{2}+M^{2}}{2}-\frac{M}{\theta-m(\xi)}\right)\right. \\
& +\mu\left(\frac{e^{-(\theta-m(\xi))(\mu-T)}}{\theta-m(\xi)}-T\right)-\left(\frac{S_{1}}{\alpha}-\frac{1}{(\theta-m(\xi))^{2}}\right) \\
& \left.\left(e^{-(\theta-m(\xi)) \mu}-e^{-(\theta-m(\xi)) M}\right)\right\} f(x) d x .
\end{aligned}
$$

The cost function comprised with expected total revenue (ETR) consisting with sale revenue and opportunity gain, and expected total cost (ETC) consisting with opportunity loss, ordering cost, purchasing cost, holding cost, deterioration cost, backlogging cost, lost sale and preservation 
technology cost. For this case, we denote expected profit function as $\operatorname{ETP}_{1,1}(T, \xi)$

$$
\begin{aligned}
E T P_{1,1}(T, \xi)= & E T R-E T C \\
= & s \overline{V_{1}}+s e I_{e 1}^{-}-A-p \overline{Q_{1}}-h \overline{I_{1}}-d \overline{D_{1}}-b \overline{B_{1}} \\
& -l \overline{L_{1}}-p c I_{c 1}^{-}-\xi \bar{P}
\end{aligned}
$$

\subsection{Case $0 \leq \mu<M \leq T$}

The random opportunity gain and opportunity cost are as follows:

$$
I_{e 2}=\left\{\begin{array}{rr}
\int_{0}^{x} \int_{0}^{t} \alpha \eta d \eta d t+(M-x) \int_{0}^{x} \alpha t d t, & 0 \leq x \leq \mu ; \\
\int_{0}^{\mu} \int_{0}^{t} \alpha \eta d \eta d t+\int_{\mu}^{M} \int_{0}^{\mu} \alpha \eta d \eta d t & \\
+\int_{\mu}^{x} \int_{\mu}^{t} \alpha \mu d \eta d t+(M-x) \int_{\mu}^{x} \alpha \mu d t, & \mu \leq x \leq M \\
\int_{0}^{\mu} \int_{0}^{t} \alpha \eta d \eta d t+\int_{\mu}^{x} \int_{0}^{\mu} \alpha \eta d \eta d t & \\
+\int_{\mu}^{x} \int_{\mu}^{t} \alpha \mu d \eta d t, & M \leq x \leq T ; \\
\int_{0}^{\mu} \int_{0}^{t} \alpha \eta d \eta d t+\int_{\mu}^{T} \int_{0}^{\mu} \alpha \eta d \eta d t & \\
+\int_{\mu}^{T} \int_{\mu}^{t} \alpha \mu d \eta d t, & x \geq T ;
\end{array}\right.
$$

and

$$
I_{c 2}= \begin{cases}0, & 0 \leq x \leq \mu \\ 0, & \mu \leq x \leq M \\ \int_{M}^{x} i_{2}(t) d t, & M \leq x \leq T \\ \int_{M}^{T} i_{2}(t) d t, & x \geq T\end{cases}
$$

Hence, the expected values of opportunity gain and opportunity cost are as follows:

$$
\begin{aligned}
I_{e 2}^{-}=E\left(I_{e 2}\right)= & \alpha \int_{x_{\min }}^{\mu}\left(\frac{M}{2}-\frac{x}{3}\right) x^{2} f(x) d x \\
& +\alpha \mu \int_{\mu}^{M}\left\{\left(\frac{M}{2}-\frac{\mu}{3}\right) \mu\right. \\
& \left.+(x-\mu)\left(M-\frac{\mu+x}{2}\right)\right\} f(x) d x \\
& +\frac{\alpha \mu}{2} \int_{M}^{T}\left(\frac{\mu^{2}}{3}-\mu x+x^{2}\right) f(x) d x \\
& +\frac{\alpha \mu}{2} \int_{T}^{x_{\max }}\left(\frac{\mu^{2}}{3}-\mu T+T^{2}\right) f(x) d x
\end{aligned}
$$

and

$$
\begin{aligned}
I_{c 2}^{-}=E\left(I_{c 2}\right)= & -\frac{\alpha \mu}{\theta-m(\xi)} \\
& \times \int_{M}^{T}\left(\frac{e^{-(\theta-m(\xi))(x-T)}-e^{-(\theta-m(\xi))(M-T)}}{\theta-m(\xi)}\right. \\
& +x-M) f(x) d x+\frac{\alpha \mu}{\theta-m(\xi)} \\
& \int_{T}^{x_{\max }}\left(\frac{e^{-(\theta-m(\xi))(M-T)}-1}{\theta-m(\xi)}\right. \\
& -T+M) f(x) d x .
\end{aligned}
$$

If we denote $\operatorname{ETP}_{1,2}$ be the expected profit function for this case, then

$$
\begin{aligned}
\operatorname{ETP}_{1,2}(T, \xi)= & E T R-E T C \\
= & s \overline{V_{1}}+s e \overline{I_{e 2}}-A-p \overline{Q_{1}}-h \overline{I_{1}}-d \overline{D_{1}}-b \overline{B_{1}} \\
& -l \overline{L_{1}}-p c \overline{I_{c 2}}-\xi \bar{P} .
\end{aligned}
$$

\subsection{Case $0 \leq \mu \leq T<M$}

In this case $T<M$. Hence, there is no interest charge on the retailer. The random opportunity gain and opportunity cost for this case are:

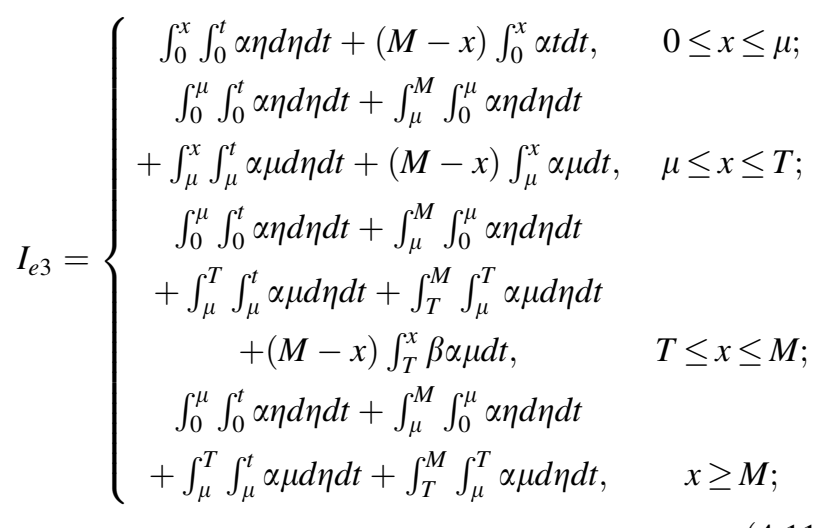

and

$$
I_{c 3}=0
$$

The expected values of opportunity gain and opportunity cost are as follows: 


$$
\begin{aligned}
I_{e 3}^{-}=E\left(I_{e 3}\right)= & \alpha \int_{x_{\min }}^{\mu}\left(\frac{M}{2}-\frac{x}{3}\right) x^{2} f(x) d x \\
& +\alpha \mu \int_{\mu}^{T}\left\{\left(\frac{M}{2}-\frac{\mu}{3}\right) \mu\right. \\
& \left.+(x-\mu)\left(M-\frac{\mu+x}{2}\right)\right\} f(x) d x \\
& +\alpha \mu \int_{T}^{M}\left\{\left(\frac{M}{2}-\frac{\mu}{3}\right) \mu\right. \\
& +(T-\mu)\left(M-\frac{\mu+T}{2}\right) \\
& +\beta(M-x)(x-T)\} f(x) d x \\
& +\alpha \mu \int_{M}^{x_{\max }}\left\{\left(\frac{M}{2}-\frac{\mu}{3}\right) \mu\right. \\
& \left.+(T-\mu)\left(M-\frac{\mu+T}{2}\right)\right\} f(x) d x \\
& I_{c 3}^{-}=E\left(I_{c 3}\right)=0 .
\end{aligned}
$$

If we denote expected total profit for this case as $E T P_{1,3}$, then

$$
\begin{aligned}
\operatorname{ETP}_{1,3}(T)= & E T R-E T C \\
= & s \overline{V_{1}}+s e \overline{I_{e 3}}-A-p \overline{Q_{1}}-h \overline{I_{1}}-d \overline{D_{1}}-b \overline{B_{1}} \\
& -l \overline{L_{1}}-p c \overline{I_{c 3}}-\xi \bar{P} .
\end{aligned}
$$

\subsection{Case $0 \leq M \leq T \leq \mu$}

The random interest earned and interest paid for this case are obtained as follows:

$$
I_{e 4}= \begin{cases}\int_{0}^{x} \int_{0}^{t} \alpha \eta d \eta d t+(M-x) \int_{0}^{x} \alpha t d t, & 0 \leq x \leq M \\ \int_{0}^{x} \int_{0}^{t} \alpha \eta d \eta d t, & M \leq x \leq T \\ \int_{0}^{T} \int_{0}^{t} \alpha \eta d \eta d t, & x \geq T\end{cases}
$$

and

$$
I_{c 4}= \begin{cases}0, & 0 \leq x \leq M \\ \int_{M}^{x} i d t, & M \leq x \leq T \\ \int_{M}^{T} i d t, & x \geq T\end{cases}
$$

The expected values of interest earned and interest paid are as follows:

$$
\begin{aligned}
-\overline{I_{e 4}}=E\left(I_{e 4}\right)= & \alpha \int_{x_{\min }}^{M}\left(\frac{M}{2}-\frac{x}{3}\right) x^{2} f(x) d x \\
& +\alpha \int_{M}^{T} \frac{x^{3}}{6} f(x) d x+\alpha \int_{T}^{x_{\max }} \frac{T^{3}}{6} f(x) d x,
\end{aligned}
$$

$$
\begin{aligned}
I_{c 4}^{-}=E\left(I_{c 4}\right)= & -\frac{\alpha}{\theta-m(\xi)} \int_{M}^{T}\left(\left(\frac{x^{2}-M^{2}}{2}-\frac{x-M}{\theta-m(\xi)}\right)\right. \\
& \left.+\left(\frac{S_{2}}{\alpha}-\frac{1}{(\theta-m(\xi))^{2}}\right)\left(e^{-(\theta-m(\xi)) x}-e^{-(\theta-m(\xi)) M}\right)\right) f(x) d x \\
& -\frac{\alpha}{\theta-m(\xi)} \int_{T}^{x_{\max }}\left\{\left(\frac{T^{2}-M^{2}}{2}-\frac{T-M}{\theta-m(\xi)}\right)\right. \\
& \left.+\left(\frac{S_{2}}{\alpha}-\frac{1}{(\theta-m(\xi))^{2}}\right)\left(e^{-(\theta-m(\xi)) T}-e^{-(\theta-m(\xi)) M}\right)\right\} \\
& f(x) d x .
\end{aligned}
$$

If we denote $E T P_{2,1}$ as the expected total profit for this case, then

$$
\begin{aligned}
& E_{2,1}(T, \xi)=s \overline{V_{2}}+s e \overline{I_{e 4}}-A-p \overline{Q_{2}}-h \overline{I_{2}} \\
& -d \overline{D_{2}}-b \overline{B_{2}}-l \overline{L_{2}}-p c \overline{I_{c 4}}-\xi \bar{P} .
\end{aligned}
$$

\subsection{Case $0 \leq T<M \leq \mu$}

In this case $T<M$, hence, no interest is charged on the retailer. Hence, random interest earn by the retailer is

$$
I_{e 5}=\left\{\begin{array}{cc}
\int_{0}^{x} \int_{0}^{t} \alpha \eta d \eta d t+(M-x) \int_{0}^{x} \alpha t d t, & 0 \leq x \leq T \\
\int_{0}^{T} \int_{0}^{t} \alpha \eta d \eta d t+\int_{T}^{M} \int_{0}^{T} \alpha \eta d \eta d t & \\
+(M-x) \int_{T}^{x} \beta \alpha t d t, & T \leq x \leq M \\
\int_{0}^{T} \int_{0}^{t} \alpha \eta d \eta d t+\int_{T}^{M} \int_{0}^{T} \alpha \eta d \eta d t, & x \geq M
\end{array}\right.
$$

and interest paid is

$$
I_{c 5}=0 \text {. }
$$

Hence, the expected values of interest terms are as follows:

$$
\begin{aligned}
I_{e 5}^{-}=E\left(I_{e 5}\right)= & \alpha \int_{x_{\min }}^{T}\left(\frac{M}{2}-\frac{x}{3}\right) x^{2} f(x) d x \\
& +\alpha \int_{T}^{M}\left\{\left(\frac{M}{2}-\frac{T}{3}\right) T^{2}\right. \\
& \left.+\beta(M-x) \frac{x^{2}-T^{2}}{2}\right\} f(x) d x \\
& +\alpha \int_{M}^{x_{\max }}\left(\frac{M}{2}-\frac{T}{3}\right) T^{2} f(x) d x
\end{aligned}
$$

and

$$
I_{c 5}^{-}=E\left(I_{c 5}\right)=0 \text {. }
$$

If we denote $E T P_{2,2}$ as the expected total profit for this case, then

$$
\begin{aligned}
& E_{2,2}(T, \xi)=s \overline{V_{2}}+s e \overline{I_{e 5}}-A-p \overline{Q_{2}}-h \overline{I_{2}} \\
& -d \overline{D_{2}}-b \overline{B_{2}}-l \overline{L_{2}}-p c \overline{I_{c 5}}-\xi \bar{P} .
\end{aligned}
$$




\subsection{Case $0 \leq T<\mu \leq M$}

In this case also there is no interest charged on the retailer, only earns. The random interest terms are as follows:

$$
I_{e 6}=\left\{\begin{array}{cc}
\int_{0}^{x} \int_{0}^{t} \alpha \eta d \eta d t+(M-x) \int_{0}^{x} \alpha t d t, & 0 \leq x \leq T ; \\
\int_{0}^{T} \int_{0}^{t} \alpha \eta d \eta d t+\int_{T}^{M} \int_{0}^{T} \alpha \eta d \eta d t & \\
+(M-x) \int_{T}^{x} \beta \alpha t d t, & T \leq x \leq \mu ; \\
\int_{0}^{T} \int_{0}^{t} \alpha \eta d \eta d t+\int_{T}^{M} \int_{0}^{T} \alpha \eta d \eta d t & \\
+\int_{x}^{M} \int_{T}^{\mu} \beta \alpha \eta d \eta d t & \\
+(M-x) \int_{\mu}^{x} \beta \alpha \mu d t, & \mu \leq x \leq M ; \\
\int_{0}^{T} \int_{0}^{t} \alpha \eta d \eta d t+\int_{T}^{M} \int_{0}^{T} \alpha \eta d \eta d t, & x \geq M ;
\end{array}\right.
$$

and the interest paid is

$$
I_{c 6}=0 .
$$

Expected values of interest terms are as follows:

$$
\begin{aligned}
I_{e 6}^{-}=E\left(I_{e 6}\right)= & \alpha \int_{x_{\min }}^{T}\left(\frac{M}{2}-\frac{x}{3}\right) x^{2} f(x) d x \\
& +\alpha \int_{T}^{\mu}\left\{\left(\frac{M}{2}-\frac{T}{3}\right) T^{2}\right. \\
& \left.+\beta(M-x) \frac{x^{2}-T^{2}}{2}\right\} f(x) d x \\
& +\alpha \int_{\mu}^{M}\left\{\left(\frac{M}{2}-\frac{T}{3}\right) T^{2}\right. \\
& \left.-\frac{\beta}{2}(M-x)\left(T^{2}-2 \mu x+\mu^{2}\right)\right\} f(x) d x \\
& +\alpha \int_{M}^{x_{\max }}\left(\frac{M}{2}-\frac{T}{3}\right) T^{2} f(x) d x,
\end{aligned}
$$$$
I_{c 6}^{-}=E\left(I_{c 6}\right)=0 .
$$

If we denote the expected total profit as $E T P_{2,3}$, then

$$
\begin{gathered}
E T P_{2,3}(T, \xi)=s \overline{V_{2}}+s e \overline{I_{e 6}}-A-p \overline{Q_{2}} \\
-h \overline{I_{2}}-d \overline{D_{2}}-b \overline{B_{2}}-l \overline{L_{2}}-p c I_{c 6}^{-}-\xi \bar{P} .
\end{gathered}
$$

\section{Model I: When $M \leq \mu$}

Cases $4.1,4.4$ and 4.5 satisfy the condition $M \leq \mu$. Hence, integrated cost function for this model is comprised with Eqs. (4.5), (4.20) and (4.25). If we denote the integrated cost by $E T P_{1}$, then

$$
E P_{1}= \begin{cases}E T P_{1,1}, & M \leq \mu \leq T ; \\ E T P_{2,1}, & M \leq T \leq \mu ; \\ E T P_{2,2}, & T \leq M \leq \mu .\end{cases}
$$

\subsection{Solution procedure}

The cost function is a profit function. Hence, our aim is to maximize it under time and preservation technology cost constraints as follows:

$$
\left\{\begin{array}{l}
\text { Maximize : } \operatorname{ETP}_{1}(T, \xi) \\
\text { subjectto : } \\
0<T<x_{\max } \\
0<\xi<W .
\end{array}\right.
$$

Each component of $E T P_{1}$ has an optimal policy which maximizes the profit, but is local. In order to find the unique optimum policy, we here write a procedure.

\section{Algorithms 1}

Step 1. Maximize $E T P_{1,1}$ subject to the constraints $0<T<x_{\max }, 0<\xi<W$ and $\mu \leq T$ by using the command FindMaximium in Mathematica.

Step 2. Maximize $E T P_{2,1}$ subject to the constraints $0<T<x_{\max }, 0<\xi<W$ and $M \leq T \leq \mu$ by using the command FindMaximium in Mathematica.

Step 3. Maximize $E_{T P_{2,2}}$ subject to the constraints $0<T<x_{\max }, 0<\xi<W$ and $T \leq M$ by using the command FindMaximium in Mathematica.

Step 4. Set $\left(i^{*}, j^{*}\right)=\arg \left(\max _{i, j \in\{1,2\}} E T P_{i, j}^{*}\right)$, where $E T P_{i, j}^{*}$ is the optimal value of $E T P_{i, j}$.

Step 5. Optimal Solution is, ETP $P_{1}^{*}=E T P_{i^{*}, j^{*}}, T=T_{i^{*}, j^{*}}$ and $\xi^{*}=\xi_{i^{*}, j^{*}}$.

\section{Model II: When $\mu<M$}

Since the cases $4.2,4.3$ and 4.6 satisfy the condition $\mu<M$. Hence the integrated cost function for this model is consisted with Eqs. (4.10), (4.15) and (4.30) as follows. If we denote the integrated profit by $\operatorname{ETP}_{2}(T, \xi)$, then

$$
\operatorname{ETP}_{2}(T, \xi)= \begin{cases}E T P_{1,2}, & \mu \leq M \leq T \\ E T P_{1,3}, & \mu \leq T \leq M \\ E T P_{2,3}, & T \leq \mu \leq M\end{cases}
$$

\subsection{Solution procedure}

Similar to Model I, our aim is to maximize the profit function $\operatorname{ETP}_{2}(T, \xi)$ subject to the constraints $0<T<x_{\max }, 0<\xi<W$. The following procedures give the unique optimal policy.

\section{Algorithms 2}

Step 1. Maximize $E T P_{1,2}$ subject to the constraints $0<T<x_{\max }, 0<\xi<W$ and $M \leq T$ by using the command FindMaximium in Mathematica. 


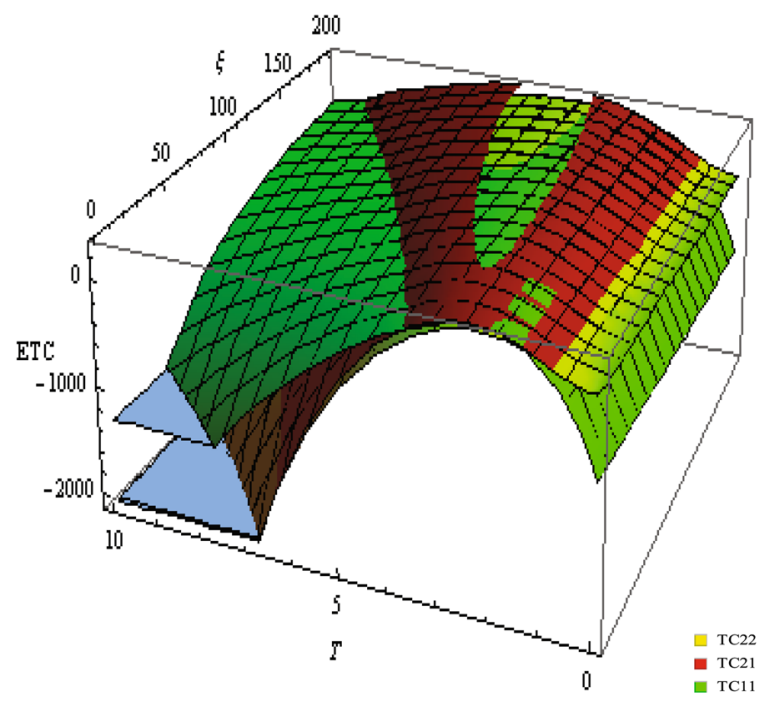

(a) $M \leq \mu$

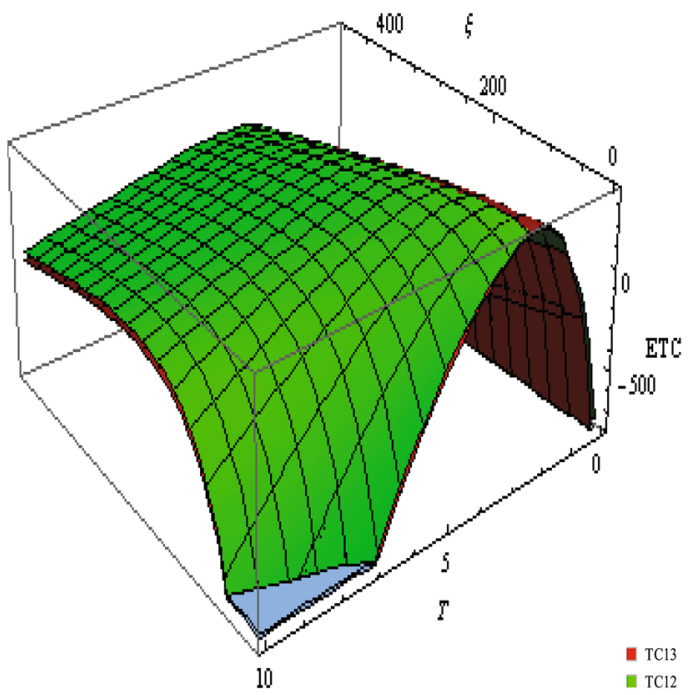

(b) $\mu<M$

Figure 2. Concavity of profit function for exponentially distributed planning horizon.

Step 2. Maximize $E T P_{1,3}$ subject to the constraints $0<T<x_{\max }, 0<\xi<W$ and $\mu \leq T \leq M$ by using the command FindMaximium in Mathematica.

Step 3. Maximize $E T P_{2,3}$ subject to the constraints $0<T<x_{\max }, 0<\xi<W$ and $T \leq \mu$ by using the command FindMaximium in Mathematica.

Step 4. Set $\left(i^{*}, j^{*}\right)=\arg \left(\max _{i, j \in\{1,2,3\}} E T P_{i, j}^{*}\right)$, where $E T P_{i, j}^{*}$ is the maximum value of $E T P_{i, j}$.

Step 5. Optimal Solution is, $E T P_{2}^{*}=E T P_{i^{*}, j^{*}}, T=T_{i^{*}, j^{*}}$ and $\xi^{*}=\xi_{i^{*}, j^{*}}$.

\section{Illustrative example}

In this section, we present some numerical examples to illustrate the mathematical formulation. We also carry out sensitivity analysis by changing the value of key parameters to test the robustness of the model, and to delineate the managerial insights also.

Example 1 Let us consider an inventory problem with input parameters in appropriate units as $\alpha=25, \beta=0.25$, $\gamma=0.01, \theta=0.2, A=120, W=500, s=40, p=10$, $h=0.05, d=0.1, b=2, l=3, e=0.09, c=0.1, M=1$, $\mu=2$. Again let us consider that the planning horizon is exponentially distributed with mean $\delta=1$, i.e., the probability density function of planning horizon is $f(x)=e^{-\delta x}, 0 \leq x \leq \infty$.

Since $M<\mu$, Model I will give the optimal policy. Using the Algorithm 1, we find the optimal policy as follows: $\left(i^{*}, j^{*}\right)=(1,1), E T P_{1}^{*}=E T P_{1,1}=319.142, \xi^{*}=61.6252$, $T^{*}=2.74043, Q^{*}=28.458$.

If $M=2.5, \mu=2$, then optimal policy can be obtained from Model II as follows: $\left(i^{*}, j^{*}\right)=(1,2)$,
$E_{T P}^{*}=E_{2} P_{1,2}=372.666, \quad \xi^{*}=74.0785, T^{*}=2.96985$, $Q^{*}=29.554$. Concavity of the profit functions for given data set are shown in figure 2, that ensure the uniqueness of unique optimal policy.

Sensitivity analysis. The effect of key parameters $\theta, \beta$, $\gamma, p, e, c, M, \mu$ and $\delta$ on the optimal policy are examined by changing its values as $-50 \%,-25 \%,+25 \%$ and $+50 \%$, respectively. The effects on the both models for this changes are shown in table 1, figure 3 and figure 4 . When $\theta$ is increased, $\xi$ and ETP increase, while $Q$ is almost constant for both the models. $\xi$ increases because for larger deterioration rate. Thus one has to invest more amount in preservation technology. When $\gamma$ is increases, $m(\xi)$ i.e., reduction in deterioration rate increases, consequently, ETP increases and $Q$ decreases. This improvement in optimal policy is achieved by increasing $\xi$. Sensitivity analysis indicates that $\xi$ is significantly affected by others parameters, also. Increasing value of $\beta$ increases ETP in both the models because of larger $\beta$ increases the sale revenue. Both the figures indicate that mean value $\delta$ of exponentially distributed planning horizon is highly sensitive. It is because, for a longer planning horizon (i.e., longer business span), one can earn more profit. Case $\mu<M$ is shown in figure 4. It indicates that $\mu$ is highly sensitive. Table 1 indicates that model $M \leq \mu$ provides more profit compare to model $\mu<M$. It is because, for longer $\mu$, demand rate linearly increases rapidly, consequently, the total demand for the planning horizon increases, hence, provides more profit. The dots ' - ' in both the tables indicate infeasible solutions.

The other observations for cost parameters are straightforward which ensure the robustness of the mathematical formulation. 
Table 1. Sensitivity analysis under variation of various parameter for exponential distribution.

\begin{tabular}{|c|c|c|c|c|c|c|}
\hline Parameter & \%change & $\left(i^{*}, j^{*}\right)$ & $\xi^{*}$ & $\bar{T}^{*}$ & $E \bar{T} P^{*}$ & $\bar{Q}^{*}$ \\
\hline \multicolumn{7}{|l|}{$\overline{M \leq \mu}$} \\
\hline \multirow[t]{4}{*}{$\theta$} & -50 & $(1,1)$ & 0.000056 & 2.78897 & 388.237 & 28.229 \\
\hline & -25 & $(1,1)$ & 32.857 & 2.74043 & 347.91 & 28.458 \\
\hline & 25 & $(1,1)$ & 83.9395 & 2.74043 & 296.828 & 28.458 \\
\hline & 50 & $(1,1)$ & 102.172 & 2.74043 & 278.595 & 28.4579 \\
\hline \multirow[t]{4}{*}{$\beta$} & -50 & $(1,1)$ & 71.0183 & 2.89487 & 307.582 & 28.7735 \\
\hline & -25 & $(1,1)$ & 66.6283 & 2.82119 & 313.139 & 28.6206 \\
\hline & 25 & $(1,1)$ & 55.8094 & 2.65068 & 325.678 & 28.2826 \\
\hline & 50 & $(1,1)$ & 48.856 & 2.54898 & 332.869 & 28.0899 \\
\hline \multirow[t]{4}{*}{$\gamma$} & -50 & $(1,1)$ & 0.000018 & 2.43737 & 304.363 & 32.3647 \\
\hline & -25 & $(1,1)$ & 25.4241 & 2.43831 & 305.748 & 29.5386 \\
\hline & 25 & $(1,1)$ & 72.0177 & 2.9101 & 334.242 & 27.6102 \\
\hline & 50 & $(1,1)$ & 74.5207 & 3.02241 & 347.663 & 26.9873 \\
\hline \multirow[t]{4}{*}{$p$} & -50 & $(1,1)$ & 36.8533 & 3.21806 & 490.791 & 37.5369 \\
\hline & -25 & $(1,1)$ & 52.6074 & 2.95839 & 398.934 & 31.8537 \\
\hline & 25 & $(1,1)$ & 66.2725 & 2.54763 & 247.339 & 26.0646 \\
\hline & 50 & $(1,1)$ & 67.4065 & 2.36958 & 181.551 & 24.1904 \\
\hline \multirow[t]{4}{*}{$e$} & -50 & $(1,1)$ & 52.5794 & 2.6027 & 295.81 & 27.8464 \\
\hline & -25 & $(1,1)$ & 57.1936 & 2.67165 & 307.297 & 28.1554 \\
\hline & 25 & $(1,1)$ & 65.8742 & 2.8088 & 331.334 & 28.7536 \\
\hline & 50 & $(1,1)$ & 69.9421 & 2.87656 & 343.86 & 29.042 \\
\hline \multirow[t]{4}{*}{$c$} & -50 & $(1,1)$ & 68.3388 & 2.8591 & 328.044 & 29.0234 \\
\hline & -25 & $(1,1)$ & 64.9253 & 2.79768 & 323.47 & 28.7319 \\
\hline & 25 & $(1,1)$ & 58.4254 & 2.68683 & 315.04 & 28.1994 \\
\hline & 50 & $(1,1)$ & 55.315 & 2.63646 & 311.149 & 27.9543 \\
\hline \multirow[t]{4}{*}{$\delta$} & -50 & $(1,1)$ & 122.809 & 4.53269 & 1107.66 & 82.3103 \\
\hline & -25 & $(1,1)$ & 87.96 & 3.37737 & 568.688 & 44.8095 \\
\hline & 25 & $(1,1)$ & 35.0461 & 2.27721 & 182.806 & 19.8036 \\
\hline & 50 & $(2,1)$ & 25.394 & 2 & 102 & 14.2583 \\
\hline \multirow[t]{4}{*}{$M$} & -50 & $(1,1)$ & 52.5818 & 2.57354 & 296.651 & 27.5207 \\
\hline & -25 & $(1,1)$ & 57.5482 & 2.66488 & 308.944 & 28.0479 \\
\hline & 25 & $(1,1)$ & 64.9606 & 2.8023 & 328.033 & 28.776 \\
\hline & 50 & $(1,1)$ & 67.6719 & 2.85246 & 336.308 & 29.0218 \\
\hline \multirow[t]{4}{*}{$\mu$} & -50 & $(1,1)$ & 4.02058 & 2.42589 & 239.165 & 22.2555 \\
\hline & -25 & $(1,1)$ & 31.7382 & 2.48221 & 284.957 & 26.4512 \\
\hline & 25 & $(1,1)$ & 84.3337 & 2.97965 & 338.895 & 29.4687 \\
\hline & 50 & $(1,1)$ & 99.6242 & 3.16494 & 347.675 & 30.0458 \\
\hline \multicolumn{7}{|l|}{$\mu<M$} \\
\hline \multirow[t]{4}{*}{$\theta$} & -50 & $(1,2)$ & 4.76382 & 2.96985 & 441.981 & 29.554 \\
\hline & -25 & $(1,2)$ & 45.3103 & 2.96985 & 401.434 & 29.554 \\
\hline & 25 & $(1,2)$ & 96.3928 & 2.96985 & 350.351 & 29.554 \\
\hline & 50 & $(1,2)$ & 114.625 & 2.96985 & 332.119 & 29.554 \\
\hline \multirow[t]{4}{*}{$\beta$} & -50 & $(1,2)$ & 82.0654 & 3.11891 & 363.449 & 29.9006 \\
\hline & -25 & $(1,2)$ & 78.3077 & 3.04752 & 367.886 & 29.7324 \\
\hline & 25 & $(1,2)$ & 69.2459 & 2.88447 & 377.851 & 29.3636 \\
\hline & 50 & $(1,2)$ & 63.6107 & 2.78927 & 383.526 & 29.158 \\
\hline \multirow[t]{4}{*}{$\gamma$} & -50 & $(1,3)$ & 0.000007 & 2.26713 & 354.085 & 29.4435 \\
\hline & -25 & $(1,2)$ & 44.7627 & 2.66123 & 354.842 & 30.7079 \\
\hline & 25 & $(1,2)$ & 81.5611 & 3.15126 & 390.199 & 28.7046 \\
\hline & 50 & $(1,2)$ & 82.3349 & 3.27363 & 405.195 & 28.0894 \\
\hline \multirow[t]{4}{*}{$p$} & -50 & $(1,2)$ & 43.4975 & 3.3886 & 539.252 & 38.4927 \\
\hline & -25 & $(1,2)$ & 62.1228 & 3.16205 & 450.54 & 32.8694 \\
\hline & 25 & $(1,2)$ & 81.9426 & 2.79983 & 301.731 & 27.2579 \\
\hline & 50 & $(1,2)$ & 86.8721 & 2.64475 & 235.838 & 25.5036 \\
\hline
\end{tabular}


Table 1 continued

\begin{tabular}{|c|c|c|c|c|c|c|}
\hline Parameter & \%change & $\left(i^{*}, j^{*}\right)$ & $\xi^{*}$ & $\bar{T}^{*}$ & $E \bar{T} P^{*}$ & $\bar{Q}^{*}$ \\
\hline \multirow[t]{4}{*}{$e$} & -50 & $(1,2)$ & 65.4551 & 2.81992 & 329.586 & 28.9084 \\
\hline & -25 & $(1,2)$ & 69.8617 & 2.89515 & 350.949 & 29.2349 \\
\hline & 25 & $(1,2)$ & 78.1057 & 3.04375 & 394.721 & 29.865 \\
\hline & 50 & $(1,2)$ & 81.9457 & 3.1166 & 417.097 & 30.1674 \\
\hline \multirow[t]{4}{*}{$c$} & -50 & $(1,2)$ & 74.8063 & 2.98307 & 372.869 & 29.6102 \\
\hline & -25 & $(1,2)$ & 74.438 & 2.97637 & 372.766 & 29.5818 \\
\hline & 25 & $(1,2)$ & 73.7276 & 2.96351 & 372.568 & 29.527 \\
\hline & 50 & $(1,2)$ & 73.3849 & 2.95734 & 372.473 & 29.5007 \\
\hline \multirow[t]{4}{*}{$\delta$} & -50 & $(1,2)$ & 135.157 & 5.00167 & 1248.15 & 87.9354 \\
\hline & -25 & $(1,2)$ & 100.597 & 3.69544 & 647.689 & 47.1826 \\
\hline & 25 & $(1,3)$ & 33.0657 & 2.25901 & 224.379 & 19.7854 \\
\hline & 50 & $(1,3)$ & 1.41192 & 2 & 136.901 & 15.868 \\
\hline \multirow[t]{4}{*}{$M$} & -50 & - & - & - & - & - \\
\hline & -25 & - & - & - & - & - \\
\hline & 25 & $(1,3)$ & 60.9195 & 2.74543 & 406.995 & 28.5797 \\
\hline & 50 & $(1,3)$ & 65.8394 & 2.8264 & 448.942 & 28.9369 \\
\hline \multirow[t]{4}{*}{$\mu$} & -50 & $(1,3)$ & 0.00007 & 2.31164 & 279.655 & 21.7397 \\
\hline & -25 & $(1,2)$ & 43.1757 & 2.67959 & 331.144 & 16.9049 \\
\hline & 25 & $(1,2)$ & 99.3254 & 3.2674 & 399.748 & 17.3603 \\
\hline & 50 & - & - & - & - & - \\
\hline
\end{tabular}

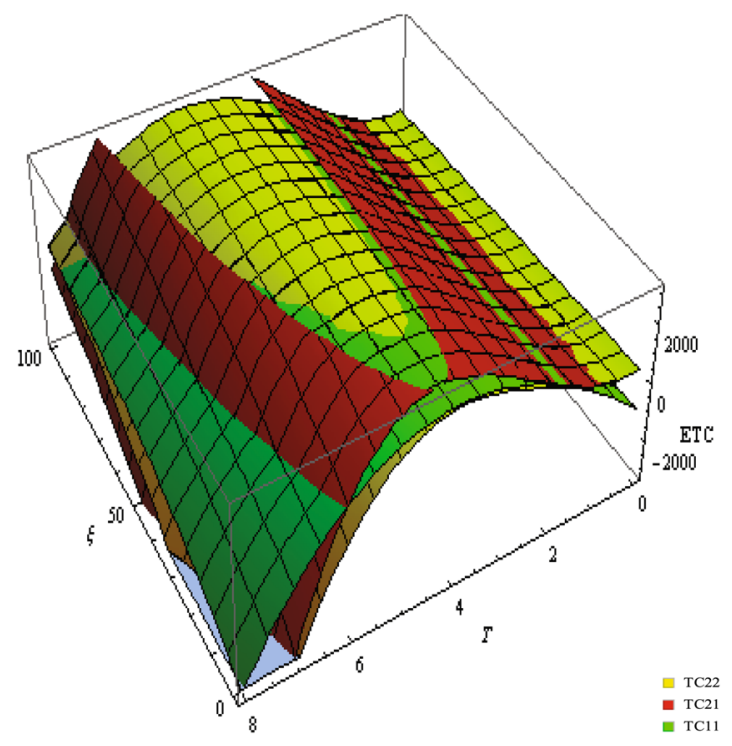

(a) $M \leq \mu$

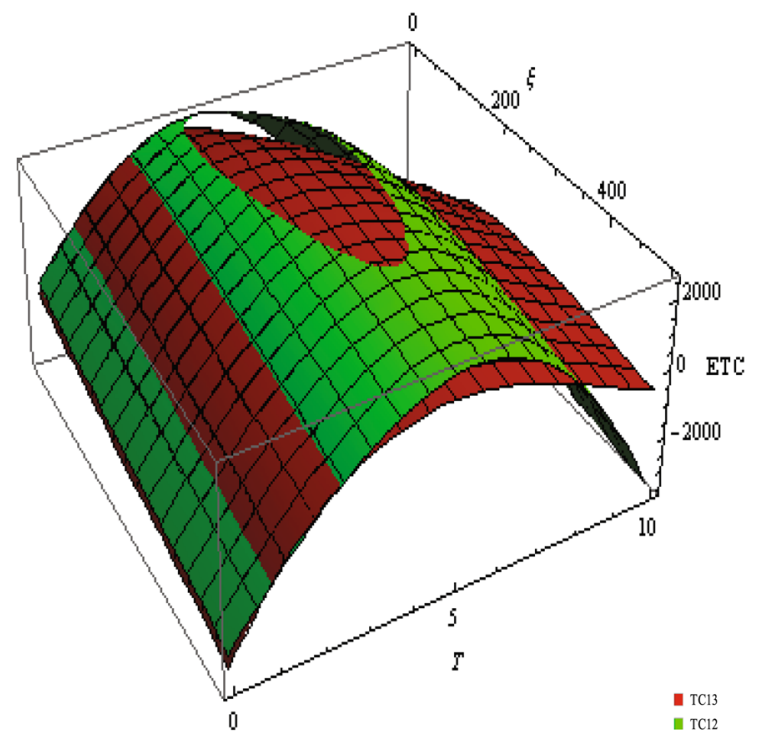

(b) $\mu<M$

Figure 3. Concavity of profit function for uniformly distributed planning horizon.

Example 2 Let us consider that the planning horizon is uniformly distributed as: $f(x)=1 / 5 ; 1 \leq x \leq 6$. The remaining data are same as of Example 1. The concavity of profit functions for given data set are shown in figure 5 .

If $M=1$ and $\mu=2$, then Model I gives the optimal policy as: $\left(i^{*}, j^{*}\right)=(1,1), \quad \operatorname{ETP}_{1}^{*}=\operatorname{ETP}_{(1,1)}=2899.89$, $\xi^{*}=132.885, T^{*}=4.94609, Q^{*}=172.431$.

If $M=2.5$ and $\mu=2$, then optimal solution from Model II is obtained as: $\left(i^{*}, j^{*}\right)=(1,2), \quad \operatorname{ETP}^{*}=3152.06$, $\xi^{*}=134.071, T^{*}=5.11944, Q^{*}=177.979$.
Sensitivity analysis. We change the parameters value same as of Example 1. The effects of changes on both the models are shown in table 2, figure 6 and figure 7. For both the models, $p$ and $\mu$ are highly sensitive, and tendency of changes are same as of Example 1. The other observations are same as of Example 1, only magnitude changes. The magnitudes of Example 2 are more than of Example 1, because means of planning horizon of Example 1 is 1 and Example 2 is 3.5. However, both the 


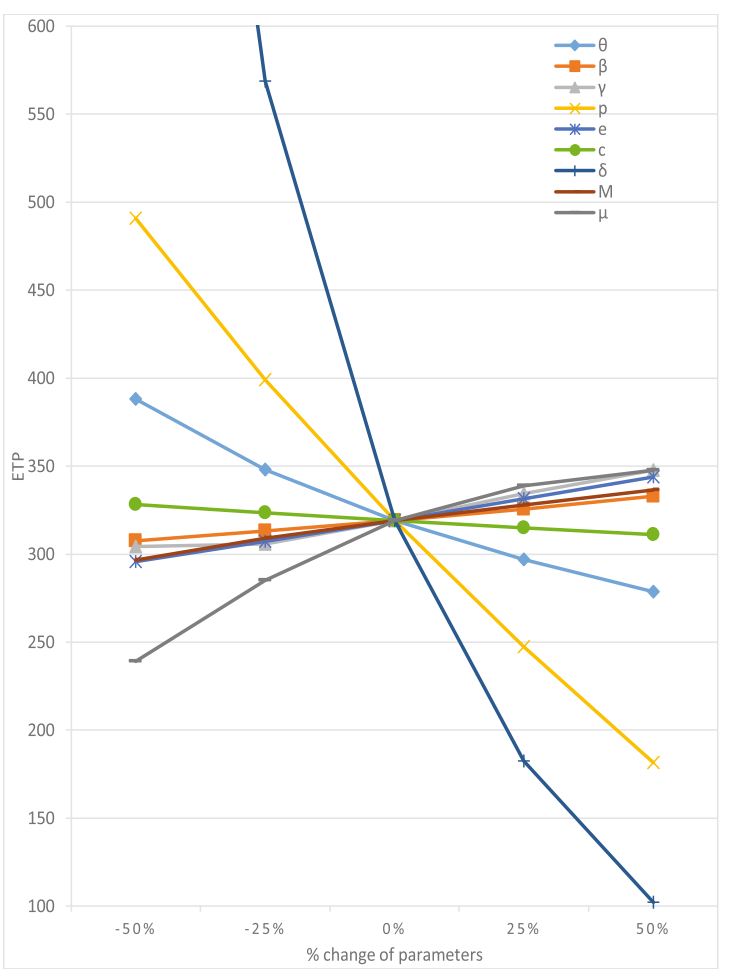

(a)

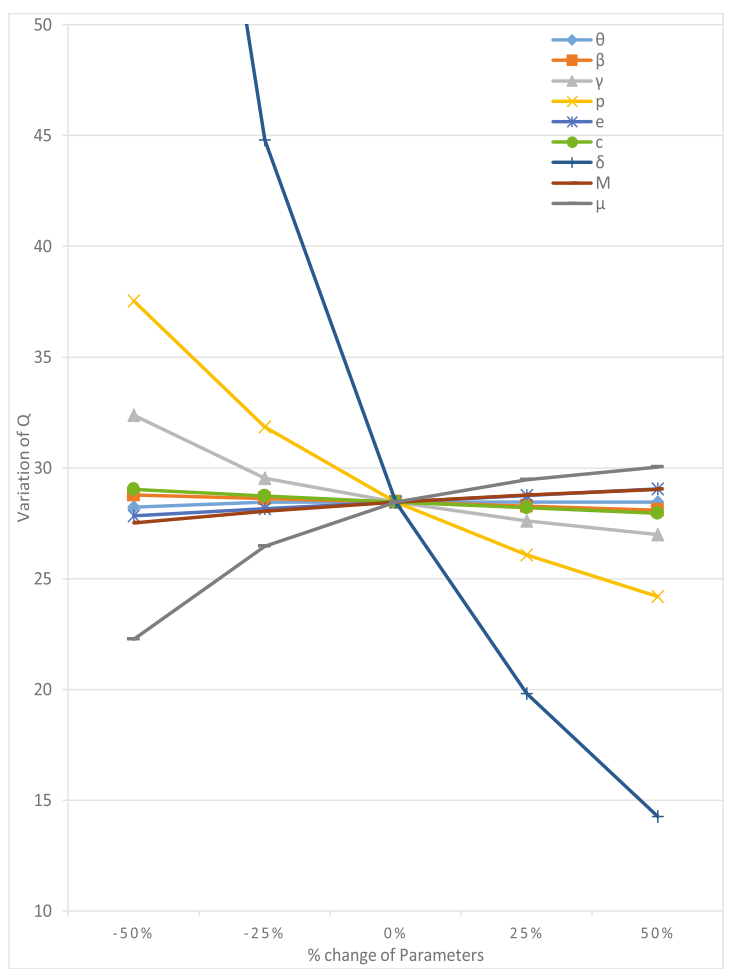

(b)

Figure 4. Effect on (a) ETP and (b) order quantity for exponentially distributed planning horizon with $M=1, \mu=2$.

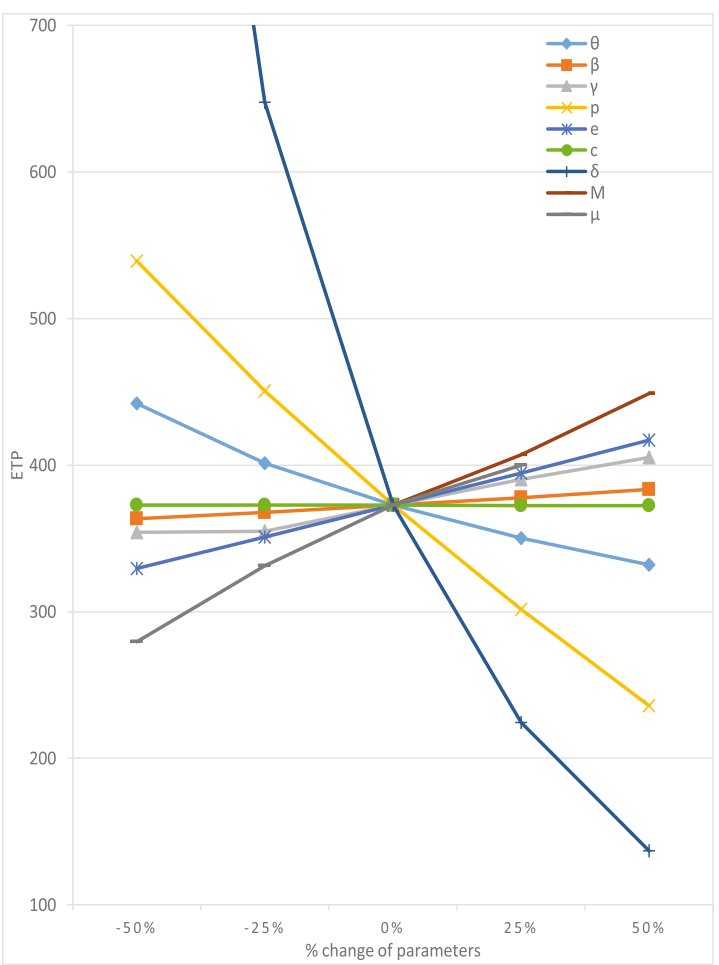

(a)

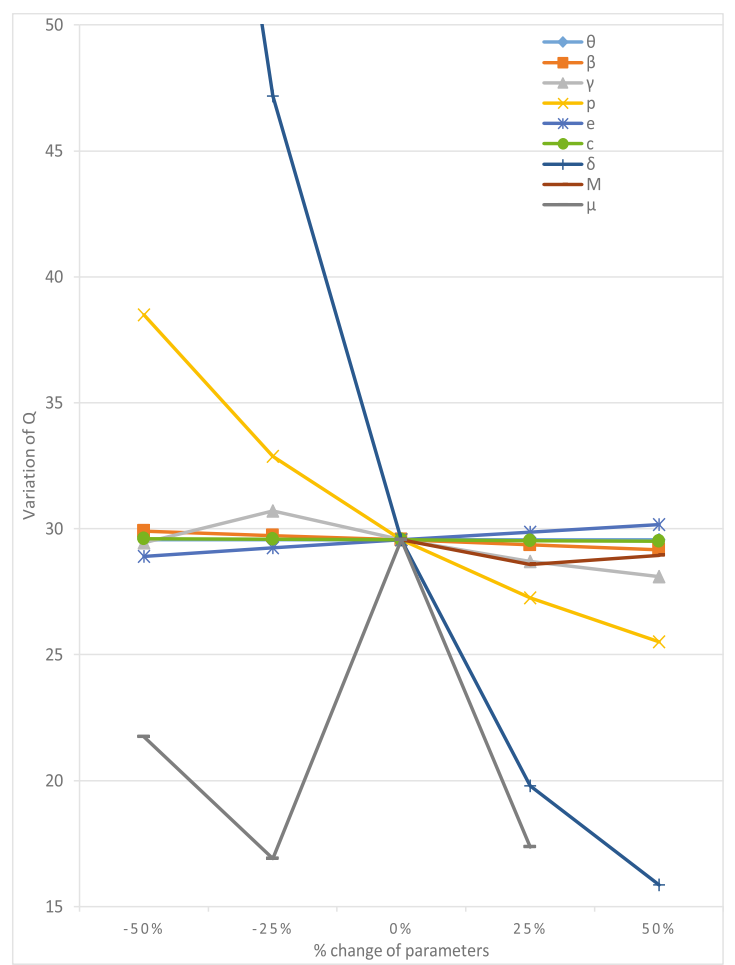

(b)

Figure 5. Effect on (a) ETP and (b) order quantity for exponentially distributed planning horizon with $M=2.5, \mu=2$. 
Table 2. Sensitivity analysis under variation of various parameter for uniform distribution.

\begin{tabular}{|c|c|c|c|c|c|c|}
\hline Parameter & \%change & $\left(i^{*}, j^{*}\right)$ & $\xi^{*}$ & $\bar{T}^{*}$ & $E \bar{T} P^{*}$ & $\bar{Q}^{*}$ \\
\hline \multicolumn{7}{|l|}{$\overline{M \leq \mu}$} \\
\hline \multirow[t]{4}{*}{$\theta$} & -50 & $(1,1)$ & 63.5701 & 4.94609 & 3142.49 & 172.431 \\
\hline & -25 & $(1,1)$ & 104.117 & 4.94609 & 3000.58 & 172.431 \\
\hline & 25 & $(1,1)$ & 155.199 & 4.94609 & 2821.79 & 172.431 \\
\hline & 50 & $(1,1)$ & 173.431 & 4.94609 & 2757.98 & 172.431 \\
\hline \multirow[t]{4}{*}{$\beta$} & -50 & $(1,1)$ & 136.522 & 5.04407 & 2879.54 & 174.041 \\
\hline & -25 & $(1,1)$ & 134.807 & 4.9976 & 2889.22 & 173.266 \\
\hline & 25 & $(1,1)$ & 130.714 & 4.88861 & 2911.71 & 171.525 \\
\hline & 50 & $(1,1)$ & 128.24 & 4.82397 & 2924.87 & 170.541 \\
\hline \multirow[t]{4}{*}{$\gamma$} & -50 & $(1,1)$ & 127.729 & 4.5754 & 2567.75 & 189.216 \\
\hline & -25 & $(1,1)$ & 139.82 & 4.83013 & 2761.95 & 178.59 \\
\hline & 25 & $(1,1)$ & 123.56 & 5.01296 & 3000.18 & 168.469 \\
\hline & 50 & $(1,1)$ & 114.704 & 5.05658 & 3076.24 & 165.715 \\
\hline \multirow[t]{4}{*}{$p$} & -50 & $(1,1)$ & 93.5289 & 5.37554 & 4005.99 & 206.472 \\
\hline & -25 & $(1,1)$ & 117.554 & 5.16626 & 3427.72 & 185.893 \\
\hline & 25 & $(1,1)$ & 142.535 & 4.71305 & 2410.19 & 161.766 \\
\hline & 50 & $(1,1)$ & 147.839 & 4.46522 & 1953.61 & 152.296 \\
\hline \multirow[t]{4}{*}{$e$} & -50 & $(1,1)$ & 124.192 & 4.72021 & 2561.65 & 167.304 \\
\hline & -25 & $(1,1)$ & 128.923 & 4.84172 & 2728.51 & 170.079 \\
\hline & 25 & $(1,1)$ & 136.217 & 5.03578 & 3074.91 & 174.428 \\
\hline & 50 & $(1,1)$ & 139.039 & 5.1131 & 3252.85 & 176.133 \\
\hline \multirow[t]{4}{*}{$c$} & -50 & $(1,1)$ & 135.794 & 5.10961 & 3058.23 & 177.068 \\
\hline & -25 & $(1,1)$ & 134.419 & 5.02789 & 2977.71 & 174.739 \\
\hline & 25 & $(1,1)$ & 131.196 & 4.86432 & 2824.76 & 170.144 \\
\hline & 50 & $(1,1)$ & 129.36 & 4.78268 & 2752.27 & 167.877 \\
\hline \multirow[t]{4}{*}{$M$} & -50 & $(1,1)$ & 133.469 & 4.87463 & 2789.1 & 169.791 \\
\hline & -25 & $(1,1)$ & 133.095 & 4.91118 & 2846.34 & 171.164 \\
\hline & 25 & $(1,1)$ & 132.823 & 4.97933 & 2949.71 & 173.594 \\
\hline & 50 & $(1,1)$ & 132.891 & 5.01086 & 2995.93 & 174.655 \\
\hline \multirow[t]{4}{*}{$\mu$} & -50 & $(1,1)$ & 83.9983 & 5.1342 & 1790.94 & 100.819 \\
\hline & -25 & $(1,1)$ & 111.693 & 4.95812 & 2383.68 & 143.674 \\
\hline & 25 & $(1,1)$ & 149.582 & 4.98317 & 3325.99 & 190.984 \\
\hline & 50 & $(1,1)$ & 163.109 & 5.04174 & 3665.03 & 201.676 \\
\hline \multicolumn{7}{|l|}{$\mu<M$} \\
\hline \multirow[t]{4}{*}{$\theta$} & -50 & $(1,2)$ & 64.7568 & 5.11944 & 3394.66 & 177.979 \\
\hline & -25 & $(1,2)$ & 105.303 & 5.11944 & 3252.75 & 177.979 \\
\hline & 25 & $(1,2)$ & 156.386 & 5.11944 & 3073.96 & 177.979 \\
\hline & 50 & $(1,2)$ & 174.618 & 5.11944 & 3010.15 & 177.979 \\
\hline \multirow[t]{4}{*}{$\beta$} & -50 & $(1,2)$ & 136.839 & 5.19942 & 3137.77 & 179.354 \\
\hline & -25 & $(1,2)$ & 135.53 & 5.16142 & 3144.58 & 178.694 \\
\hline & 25 & $(1,2)$ & 132.436 & 5.07282 & 3160.32 & 177.205 \\
\hline & 50 & $(1,2)$ & 130.587 & 5.02066 & 3169.51 & 176.362 \\
\hline \multirow[t]{4}{*}{$\gamma$} & -50 & $(1,2)$ & 135.965 & 4.79124 & 2810.85 & 196.946 \\
\hline & -25 & $(1,2)$ & 142.608 & 5.01553 & 3012.21 & 184.857 \\
\hline & 25 & $(1,2)$ & 124.084 & 5.1798 & 3252.99 & 173.576 \\
\hline & 50 & $(1,2)$ & 114.905 & 5.21932 & 3329.28 & 170.52 \\
\hline \multirow[t]{4}{*}{$p$} & -50 & $(1,2)$ & 91.5004 & 5.45893 & 4165.83 & 211.29 \\
\hline & -25 & $(1,2)$ & 116.864 & 5.2927 & 3637.32 & 190.837 \\
\hline & 25 & $(1,2)$ & 146.126 & 4.9379 & 2697.47 & 168.244 \\
\hline & 50 & $(1,2)$ & 154.446 & 4.74711 & 2268.01 & 159.989 \\
\hline \multirow[t]{4}{*}{$e$} & -50 & $(1,2)$ & 127.202 & 4.9267 & 2787.5 & 173.629 \\
\hline & -25 & $(1,2)$ & 130.953 & 5.03093 & 2968.09 & 175.994 \\
\hline & 25 & $(1,2)$ & 136.684 & 5.19491 & 3338.71 & 179.655 \\
\hline & 50 & $(1,2)$ & 138.892 & 5.25963 & 3527.49 & 181.079 \\
\hline
\end{tabular}


Table 2 continued

\begin{tabular}{|c|c|c|c|c|c|c|}
\hline Parameter & $\%$ change & $\left(i^{*}, j^{*}\right)$ & $\xi^{*}$ & $\bar{T}^{*}$ & $E \bar{T} P^{*}$ & $\bar{Q}^{*}$ \\
\hline \multirow[t]{4}{*}{$c$} & -50 & $(1,2)$ & 136.103 & 5.19562 & 3200.6 & 179.876 \\
\hline & -25 & $(1,2)$ & 135.098 & 5.15744 & 3176.04 & 178.925 \\
\hline & 25 & $(1,2)$ & 133.025 & 5.08166 & 3128.66 & 177.041 \\
\hline & 50 & $(1,2)$ & 131.961 & 5.04412 & 3105.83 & 176.109 \\
\hline \multirow[t]{4}{*}{$M$} & -50 & - & - & - & - & - \\
\hline & -25 & - & - & - & - & - \\
\hline & 25 & $(1,2)$ & 135.169 & 5.17276 & 3240.94 & 179.421 \\
\hline & 50 & $(1,2)$ & 136.263 & 5.21488 & 3339.7 & 180.472 \\
\hline \multirow[t]{4}{*}{$\mu$} & -50 & $(1,2)$ & 85.7317 & 5.34315 & 1943.03 & 102.891 \\
\hline & -25 & $(1,2)$ & 113.289 & 5.14221 & 2585.42 & 147.843 \\
\hline & 25 & $(1,2)$ & 150.35 & 5.1511 & 3626.2 & 197.467 \\
\hline & 50 & - & - & - & - & - \\
\hline
\end{tabular}

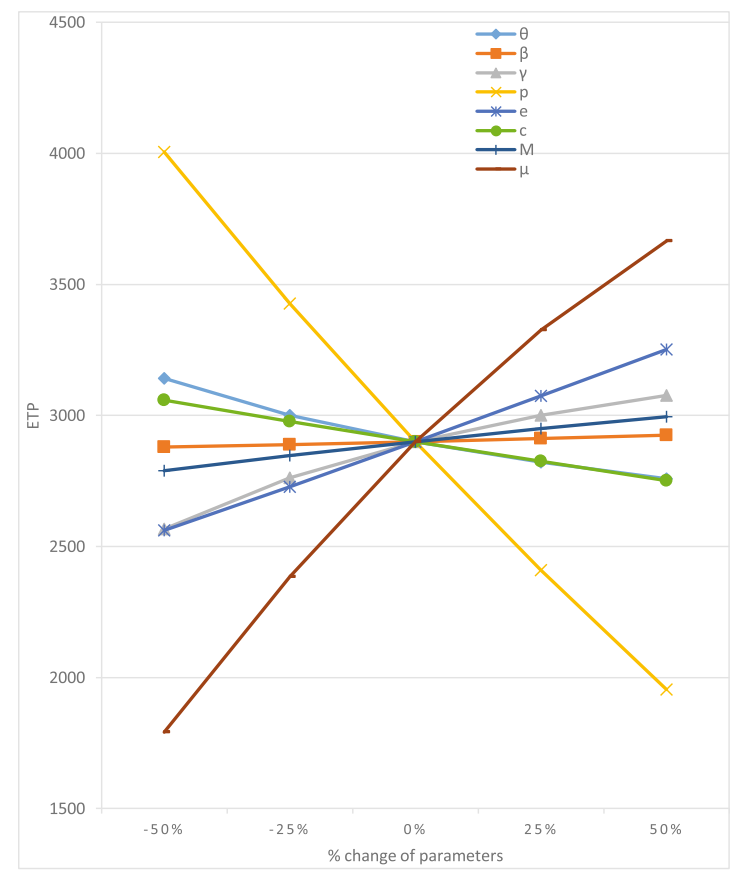

(a)

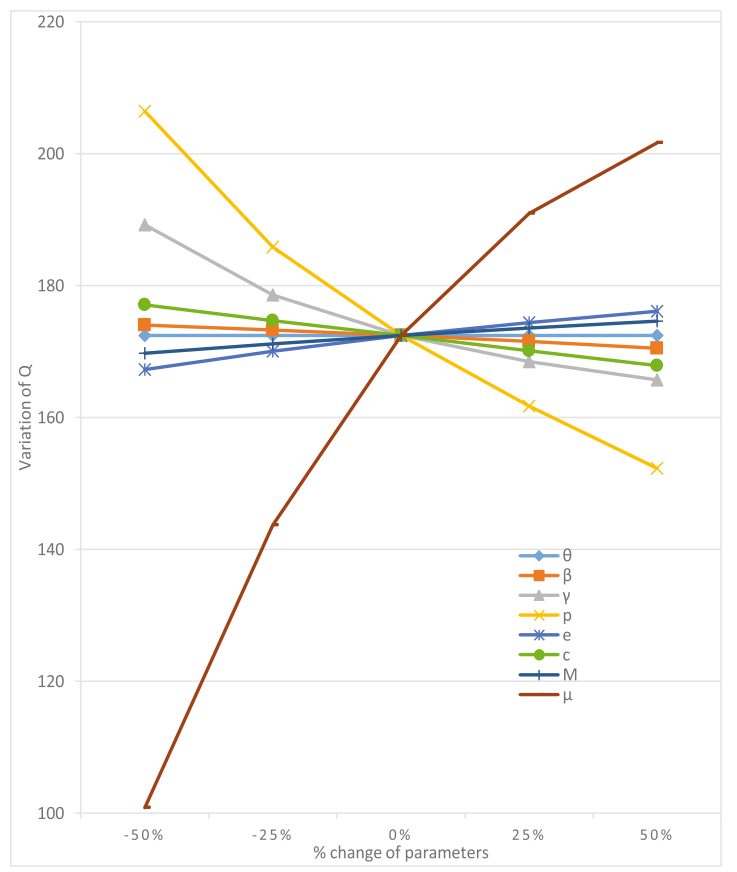

(b)

Figure 6. Effect on (a) ETP and (b) order quantity for uniformly distributed planning horizon with $M=1, \mu=2$.

examples proof the robustness of the mathematical formulation.

\section{Conclusions}

In this paper we developed a deteriorating item inventory model in the stochastic framework by integrating the concepts of preservation technology, time varying ramp-type demand and trade-credit finance in the form of permissible delay in payment. Shortage of item is allowed and is the mixture of partial backlog and lost sales. An initial investment in PT is made to reduce the deterioration rate.
The planning horizon for ramp-type demand is taken as a random variable. As per our knowledge, no inventory model under such scenario has been developed in a stochastic framework. Furthermore, the mathematical model is illustrated with some numerical examples. In the numerical experiment, random planning horizon is validated by exponential distribution and uniform distribution. The robustness of the model is tested through sensitivity analysis by changing the value of key parameters. Sensitivity analysis is also used to delineate the managerial insights.

The planning horizon of many seasonal and fashionable items randomly varies in real life business transaction. 


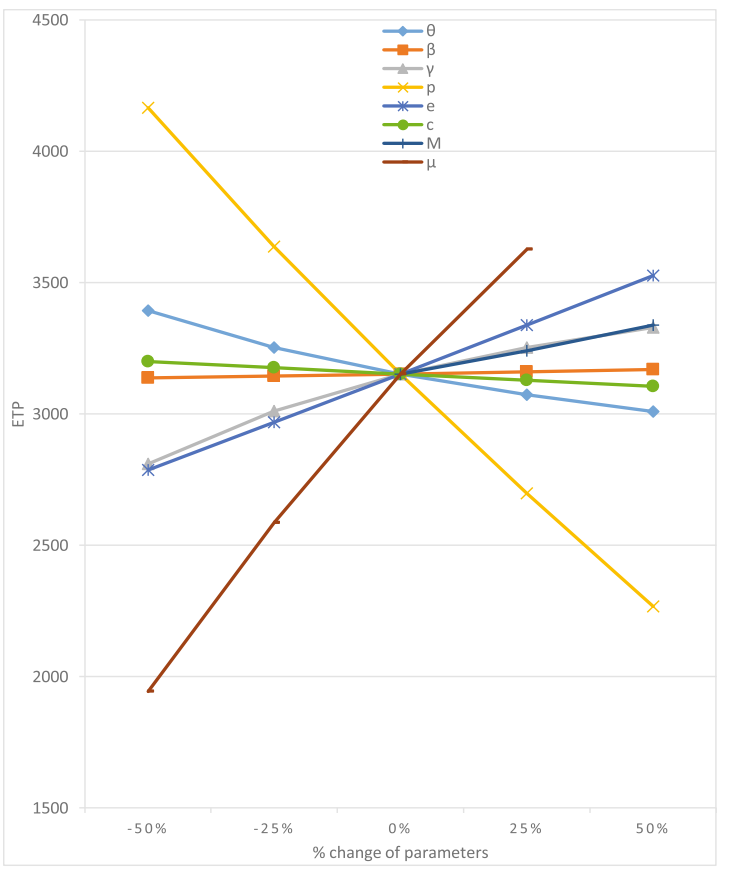

(a)

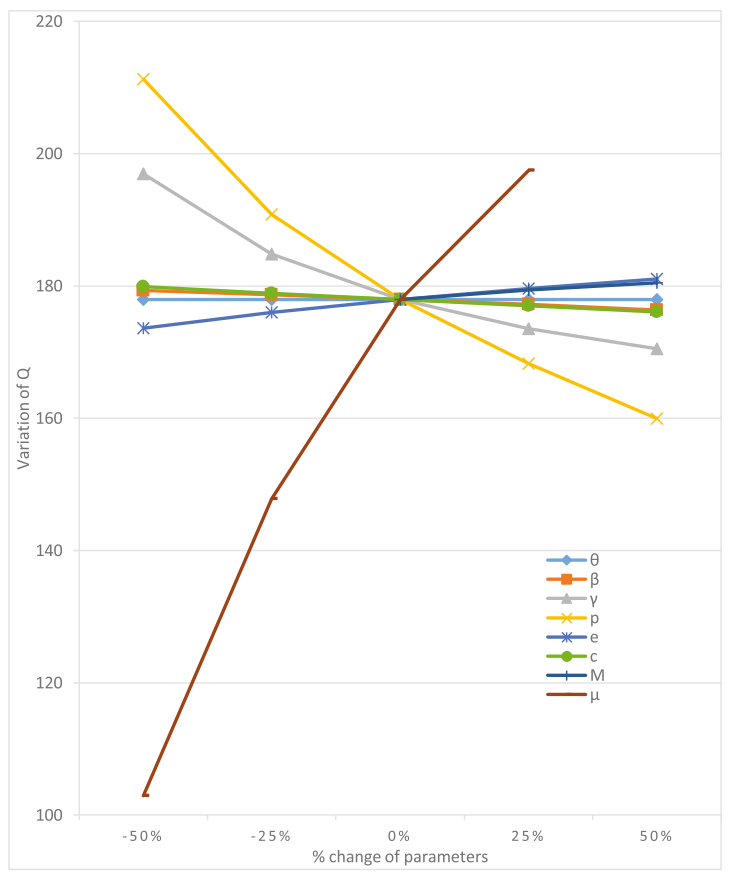

(b)

Figure 7. Effect on (a) ETP and (b) order quantity for uniformly distributed planning horizon with $M=2.5, \mu=2$.

Hence, this model can be applied while dealing with seasonal or fashionable items. In this paper, we have considered that deterioration rate is constant. But we know that deterioration rate of many items increases with time. Thus, the present model can be extended by considering nonconstant deterioration rate. The model further can be extended by considering one of the most important key parameter 'demand', as a non-deterministic variable such as random, fuzzy or fuzzy stochastic.

\section{Acknowledgements}

We express our gratefulness to the editor and the learned reviewers for their important and valuable remarks and proposals, which has led to a significant ameliorate in an earlier version of the manuscript.

\section{References}

[1] Hill R M 1995 Inventory models for increasing demand followed by level demand. J. Oper. Res. Soc. 46(10): 1250-1259

[2] De A, Mamanduru V K R, Gunasekaran A, Subramanian N, and Tiwari M K 2016 Composite particle algorithm for sustainable integrated dynamic ship routing and scheduling optimization. Comput. Ind. Eng. 96: 201-215

[3] De A, Kumar S K, Gunasekaran A and Tiwari M K 2017 Sustainable maritime inventory routing problem with time window constraints. Eng. Appl. Artif. Intell. 61: 77-95
[4] Giri B C, Jalan A K and Chaudhuri K S 2003 Economic order quantity model with Weibull deterioration distribution, shortage and ramp-type demand. Int. J. Syst. Sci. 34(4): 237-243

[5] Manna S and Chaudhuri K S 2006 An EOQ model with ramp-type demand rate, time dependent deterioration rate, unit production cost and shortages. Eur. J. Oper. Res. 171(2): 577-566

[6] Deng P S, Lin Robert H J and Chu P 2007 A note on the inventory models for deteriorating items with ramp-type demand rate . Eur. J. Oper. Res. 178(1): 112-120

[7] Panda S, Senapati S and Basu M 2008 Optimal replenishment policy for perishable seasonal products in a season with ramp-type time dependent demand. Comput. Ind. Eng. 54(2): 301-314

[8] Skouri K, Konstantaras I, Papachristos S and Teng J-T 2011 Supply chain models for deteriorating products with ramptype demand rate under permissible delay in payments. Expert Syst. Appl. 38(12): 14861-14869

[9] Kumar R S, De S K and Goswami A 2012 Fuzzy EOQ models with ramp-type demand rate, partial backlogging and time dependent deterioration rate. Int. J. Math. Oper. Res. 4(5): 473-502

[10] Kumar R S, Tiwari M K and Goswami A 2016 Two-echelon fuzzy stochastic supply chain for the manufacturerbuyer integrated productioninventory system. J. Intell. Manuf. 27(4): 875-888

[11] Kumar S and Singh A K 2016 Optimal time policy for deteriorating items of two-warehouse inventory system with time and stock dependent demand and partial backlogging. Sadhana 41(5): 541-548

[12] Kumar S, Singh A K and Patel M K 2016 Optimization of Weibull deteriorating items inventory model under the effect 
of price and time dependent demand with partial backlogging. Sadhana 41(9): 977-984

[13] Wu J, Skouri K, Teng J-T and Hu Y 2016 Two inventory systems with trapezoidal-type demand rate and time-dependent deterioration and backlogging. Expert Syst. Appl. 46: 367-379

[14] Dye C-Y and Hsieh T-P 2012 An optimal replenishment policy for deteriorating items with effective investment in preservation technology. Eur. J. Oper. Res. 218(1): 106-112

[15] Ho J C, Solis A O and Chang Y-L 2007 An evaluation of lotsizing heuristics for deteriorating inventory in material requirements planning systems. Comput. Oper. Res. 34(9): 2562-2575

[16] Ouyang L-Y, Wu K-S and Yang C-T 2006 A study on an inventory model for non-instantaneous deteriorating items with permissible delay in payments. Comput. Ind. Eng. 51(4): 637-651

[17] Hsu P H, Wee H M and Teng H M 2010 Preservation technology investment for deteriorating inventory. Int. J. Prod. Econ. 124(2): 388-394

[18] Zauberman G, Ronen R, Akerman M and Fuchs Y 1989 Low $\mathrm{pH}$ treatment protects litchi fruit color. Symp. Trop. Fruit Int. Trade 269: 309-314

[19] Tsao Y-C and Sheen G-J 2008 Dynamic pricing, promotion and replenishment policies for a deteriorating item under permissible delay in payments. Comput. Oper. Res. 35(11): 3562-3580

[20] Geetha K V and Uthayakumar R 2010 Economic design of an inventory policy for non-instantaneous deteriorating items under permissible delay in payments. J. Comput. Appl. Math. 233(10): 2492-2505

[21] Lee Y-P and Day C-Y 2012 An inventory model for deteriorating items under stock-dependent demand and controllable deterioration rate. Comput. Ind. Eng. 63(2): 474-482

[22] Hsieh T-P and Dye C-Y 2013 A production-inventory model incorporating the effect of preservation technology investment when demand is fluctuating with time. J. Comput. Appl. Math. 239: 25-36

[23] Dye C-Y 2013 The effect of preservation technology investment on a non-instantaneous deteriorating inventory model. Omega 41(5): 872-880

[24] Mishra U, Cárdenas-Barrón L E, Tiwari S, Shaikh A A and Treviño-Garza G 2017 An inventory model under price and stock dependent demand for controllable deterioration rate with shortages and preservation technology investment. Ann. Oper. Res. 254(1-2): 165-190

[25] Zhang J, Liu G, Zhang Q and Bai Z 2015 Coordinating a supply chain for deteriorating items with a revenue sharing and cooperative investment contract. Omega 56: 37-49

[26] Liu G, Zhang J and Tang W 2015 Joint dynamic pricing and investment strategy for perishable foods with price-quality dependent demand. Ann. Oper. Res. 226(1): 397-416

[27] Zhang J, Wei Q, Zhang Q and Tang W 2016 Pricing, service and preservation technology investments policy for deteriorating items under common resource constraints. Comput. Ind. Eng. 95: 1-9

[28] Goyal S K 1985 Economic order quantity under conditions of permissible delay in payments. J. Oper. Res. Soc. 36(4): 335-338

[29] Aggarwal P S and Jaggi K C 1995 Ordering policies of deteriorating items under permissible delay in payments. $J$. Oper. Res. Soc. 46(5): 658-662

[30] Chang C-T, Ouyang L-Y and Teng J-T 2003 An EOQ model for deteriorating items under supplier credits linked to ordering quantity. Appl. Math. Model. 27(12): 983-996

[31] Ouyang L-Y, Teng J-T, Goyal S K and Yang C-T 2009 An economic order quantity model for deteriorating items with partially permissible delay in payments linked to order quantity. Eur. J. Oper. Res. 194(2): 418-431

[32] Dye C-Y and Hsieh T-P 2013 A particle swarm optimization for solving lot-sizing problem with fluctuating demand and preservation technology cost under trade-credit. J. Glob. Optim. 55(3): 655-679

[33] Yang C-T, Dye C-Y and Ding J-F 2015 Optimal dynamic trade-credit and preservation technology allocation for a deteriorating inventory model. Comput. Ind. Eng. 87: 356-369

[34] Chen S-C and Teng J-T 2015 Inventory and credit decisions for time-varying deteriorating items with up-stream and down-stream trade-credit financing by discounted cash flow analysis. Eur. J. Oper. Res. 243(2): 566-575

[35] Tiwari S, Cárdenas-Barrón L E, Khanna A and Jaggi C K 2016 Impact of trade-credit and inflation on retailer's ordering policies for non-instantaneous deteriorating items in a two-warehouse environment. Int. J. Prod. Econ. 176: 154-169

[36] Tsao Y-C 2016 Joint location, inventory, and preservation decisions for non-instantaneous deterioration items under delay in payments. Int. J. Syst. Sci. 47(3): 572-585 